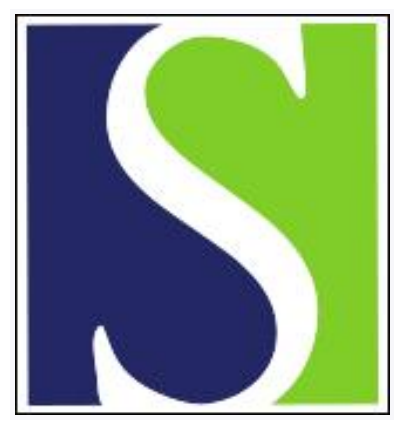

Scand J Work Environ Health 2009;35(1):19-36

https://doi.org/10.5271/sjweh.1306

Issue date: 00 Jan 2009

Associations between work-related factors and the carpal tunnel syndrome-a systematic review

by van Rijn RM, Huisstede BMA, Koes BW, Burdorf A

Affiliation: Erasmus MC, Department of General Practice, Room Wk-109, PO Box 2040, NL-3000 CA Rotterdam, Netherlands. r.vanrijn@erasmusmc.nl

Refers to the following texts of the Journal: 1998;24(4):285-292 1997;23(5):364-369 1999;25 suppl 4:25-30

The following articles refer to this text: 2009;35(5):342-348;

2010;36(1):25-33; 2010;36(1):1-2; 2010;36(3):189-201;

2010;36(5):384-393; 2010;36(6):515-516; 2011;37(2):120-128;

2011;37(4):298-306; 2013;39(2):155-163; 2013;39(5):495-505;

2013;39(6):568-577; 2016;42(4):280-290; 2017;43(2):163-170

Key terms: carpal tunnel syndrome; force; hand-arm vibration; MSD; musculoskeletal disorder; nerve compression syndrome; repetitiveness; review; systematic review; work-related factor

This article in PubMed: www.ncbi.nlm.nih.gov/pubmed/19277433 


\title{
Associations between work-related factors and the carpal tunnel syndrome-a systematic review
}

\author{
by Rogier M van Rijn, MSc, ${ }^{1}$ Bionka MA Huisstede, PhD, ${ }^{1}$ Bart W Koes, PhD, ${ }^{1}$ Alex Burdorf, PhD²
}

\begin{abstract}
van Rijn RM, Huisstede BMA, Koes BW, Burdorf A. Associations between work-related factors and the carpal tunnel syndrome-a systematic review. Scand J Work Environ Health 2009;35(1):19-36.

Objectives The aim of this study was to make a quantitative assessment of the exposure-response relationships between work-related physical and psychosocial factors and the occurrence of carpal tunnel syndrome (CTS) in occupational populations.

Methods A systematic review of the literature was conducted on the associations of type of work, physical load factors, and psychosocial aspects at work to the occurrence of CTS. The associations between work factors and CTS were expressed in quantitative measures, namely, odds ratios (OR) or relative risks.

Results Jobs with the highest risk of CTS included work in the meat- and fish-processing industry, forestry work with chain saws, and electronic assembly work (OR 76.5, 21.3, and 11.4, respectively). The occurrence of CTS was associated with high levels of hand-arm vibration, prolonged work with a flexed or extended wrist, high requirements for hand force, high repetitiveness, and their combination. No association was found between any psychosocial risk factor and CTS. Contradictory findings were reported for associations between computer work and CTS.

Conclusions This review provides consistent indications that CTS is associated with an average hand force requirement of $>4 \mathrm{~kg}$, repetitiveness at work (cycle time $<10$ seconds, or $>50 \%$ of cycle time performing the same movements), and a daily 8-hour energy-equivalent frequency-weighted acceleration of $3.9 \mathrm{~m} / \mathrm{s}^{2}$.
\end{abstract}

Key terms force, hand-arm vibration, musculoskeletal disorder, nerve compression syndrome, repetitiveness.

Hand-arm symptoms are a common problem in society, especially among the working population. Of these, carpal tunnel syndrome (CTS) is the most frequently reported neuropathy of the upper extremity (1-3). CTS results from the compromise of the median nerve function at the wrist as a result of increased pressure in the carpal tunnel (1). The clinical diagnosis of CTS is based on a history of nocturnal pins and needles, numbness, or pain in the median nerve in the innervated area of the fingers and hand, which often causes the patient to awaken at night, supported by abnormalities appearing in an electrodiagnostic examination (4). Provocation tests do not necessarily contribute to the clinical diagnosis of CTS (5).

CTS can have serious economic consequences. Feuerstein et al (6) stated that $57 \%$ of all costs associated with occupational upper-extremity disorders were due to CTS. Furthermore, Silverstein et al (7) reported an average yearly claim rate for CTS of 27.3 per 10000 full-time workers.

A review of occupational populations showed a wide range in the prevalence of CTS $(0.6-61 \%)$, the lowest prevalence occurring for industrial workers and the highest for grinders, butchers, grocery store workers, and frozen food factory workers with workers using high-force, high-repetitive gripping (8). Hagberg et al concluded that exposure to physical load (such as repetitive and forceful gripping) is a major risk factor for CTS (8). Palmer et al (9) judged that there is reasonable evidence that regular and prolonged use of handheld vibrating tools increases the risk of CTS at least twofold and that there is substantial evidence for similar or even higher risks from prolonged and highly repetitious flexion and extension of the wrist, especially when combined with a forceful grip. At the same time, the balance of evidence concerning keyboard and computer work did not indicate

1 Department of General Practice, Erasmus MC, Rotterdam, Netherlands.

2 Department of Public Health, Erasmus MC, Rotterdam, Netherlands.

Reprint requests to: Rogier M van Rijn, Erasmus MC, Department of General Practice, Room Wk-109, PO Box 2040, NL-3000 CA Rotterdam, Netherlands. [E-mail: r.vanrijn@erasmusmc.nl] 
an important association with CTS (9). Furthermore, the associations between physical risk factors (repetition, force, vibration) have been confirmed in several other reviews (10-12). The risk of developing upper-extremity symptoms is also influenced by psychosocial work characteristics. The reviews of Bongers et al (13) and Van den Heuvel et al (14) have presented evidence of an association between high job demands and low social support with upper-extremity symptoms. In addition, a review of the National Research Council concluded that high job demands and high job stress were associated with the occurrence of upper-extremity disorders (11). However, reviews on the association between psychosocial work characteristics and CTS are lacking.

The available reviews have presented overviews of the occupations in which workers are at risk of CTS and occupational risk factors for the occurrence of CTS, but they provide little guidance as to the duration and magnitude of exposure to risk factors that are associated with the development of CTS. Hence it remains a matter of debate whether it will be possible to derive exposure levels that will not increase the occurrence of CTS in occupational populations.

Therefore, this systematic review of the available evidence was conducted with the aim of providing a quantitative assessment of the exposure-response relationships between work-related physical and psychosocial factors and the occurrence of CTS in occupational populations.

\section{Methods}

\section{Literature search}

Comprehensive literature searches were conducted by the first author (RMvR) in MEDLINE (from 1966 to September 2007), EMBASE (from 1984 to September 2007), and the Cochrane Central Register of Controlled Trials (September 2007). The following keywords were used: (carpal tunnel syndrome OR median nerve) AND (work related OR physical load OR psychosocial load OR exposure) AND (association OR risk factors OR odds ratio OR relative risk). The result of the complete search strategy is available on request.

Two reviewers (RMvR and BMAH) independently selected the articles, initially based on title and abstract (figure 1). For final inclusion, the articles had to fulfill all of the following criteria: (i) the occurrence of CTS was reported in occupational populations, (ii) a quantitative description of measures of exposure or a description of a distinct exposure pattern at job level was presented, (iii) the association between work-related risk factors and CTS was expressed in a quantitative measure, such as odds ratio (OR) or relative risk (RR), or sufficient raw data were provided with which to calculate these associations, and (iv) the article was published in a peerreviewed scientific journal written in English, German, French, or Dutch. A consensus method was used to resolve disagreements.

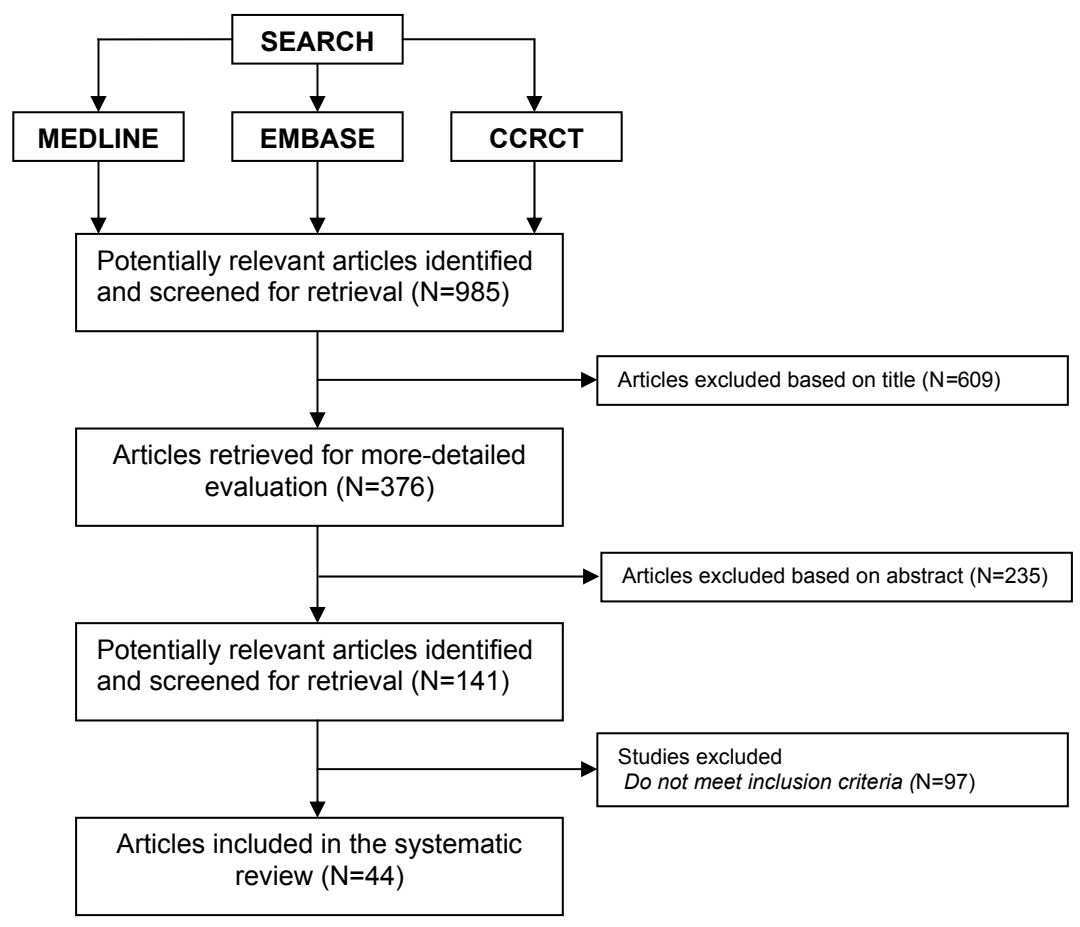

Figure 1. Flow chart of the selected articles. (CCRCT $=$ Cochrane Central Register of Controlled Trials) 


\section{Assessment of methodological quality}

A quality assessment list was constructed with the use of criteria from Huisstede et al (15), Lievense et al (16), Van Tulder et al (17) and the Dutch Cochrane Centre (18), which were adapted to the specific aim of this review (appendix I). The list covers five topics with 16 items concerning the study population, the assessment of exposure, the assessment of outcome, the study design and analysis, and the data presentation (table 1). Two reviewers ( $\mathrm{AB} \& \mathrm{BWK})$ independently assessed the quality of each study by scoring each criterion as positive, negative, or unclear. Disagreements were resolved by consensus. The quality score for every study was calculated by summing the number of positive criteria.

\section{Data extraction}

Relevant information on study population, study design, outcome ascertainment, exposure characteristics, measure of association, and confounding factors was extracted from the articles by the first author (RMvR) using a standardized form. The core findings in each article were expressed by measures of association (OR or RR value) with corresponding $95 \%$ confidence intervals $(95 \% \mathrm{CI})$. Where possible, these associations were directly extracted from the original article. For articles in which this information was not presented, associations were calculated if sufficient raw data were provided.

\section{Data analysis}

In this review three types of statistical associations were distinguished. The association was described as positive when a higher value of the risk factor was statistically associated with the occurrence of CTS. In a negative association, a higher value of the risk factor was statistically associated with a lower occurrence of CTS. In null associations, the risk estimate did not differ statistically from unity. The null associations were further evaluated as to whether the results actually suggest the absence of an effect or whether the studies were inconclusive due to a lack of information or a lack of statistical power.

First, we focused on the association between type of work (based on job descriptions with a distinct exposure pattern) and the occurrence of CTS. Second, we evaluated the associations of five types of exposure, namely, force, repetitiveness, hand-arm vibration, combined exposure measure, and awkward postures, with the occurrence of CTS. Finally, we addressed the associations between psychosocial risk factors and the occurrence of CTS.

Pooling the results of individual studies was considered only when health outcomes were clinically homogeneous, the measures of exposure were sufficiently similar according to the reviewers, and comparable study designs were used. Furthermore, the outcome of the quality assessment was used in a sensitivity analysis to evaluate whether design characteristics and the methodological quality of the studies had an impact on the reported associations between work-related risk factors and CTS.

\section{Results}

\section{Characteristics of included studies}

Our search of the literature resulted in 985 potentially relevant articles (figure 1). Of these, 44 publications met our inclusion criteria (30 cross-sectional studies, 9 case-control studies, and 5 cohort studies (appendix II). A total of 22 articles compared the occurrence of CTS across occupations (table 2), and 23 articles reported on the association between physical risk factors and CTS (table 3), of which two articles also compared the occurrence of CTS across occupations. Four articles reported on the association between psychosocial factors and CTS (table 4), of which three articles also reported on the association between physical risk factors and CTS.

\section{Methodological quality}

Table 5 presents the methodological quality assessment of the included studies. The initial agreement of the two reviewers was $68 \%$ ( 480 of 704 items). The initial disagreements were all solved in a consensus meeting.

Table 1. Methodological quality assessment.

\begin{tabular}{|c|c|c|}
\hline Criteris & & Score \\
\hline \multicolumn{3}{|c|}{ Study population } \\
\hline \multicolumn{3}{|c|}{1 Study groups (exposed and unexposed) are clearly defined $+/-/$ ? } \\
\hline \multirow{2}{*}{\multicolumn{3}{|c|}{$\begin{array}{lll}2 & \text { Participation } \geq 70 \% & +/-/ \text { ? } \\
3 & \text { Number of cases } \geq 50 & +/-/ \text { ? }\end{array}$}} \\
\hline & & \\
\hline \multicolumn{3}{|c|}{ Assessment of exposure } \\
\hline \multicolumn{3}{|c|}{ Adequate description of the exposure } \\
\hline $4 \mathrm{E}$ & Exposure definition & $+/-/ ?$ \\
\hline $5 f$ & Assessment of exposure & $+/-/ ?$ \\
\hline 6 & Blind for outcome status & $+/-1 ?$ \\
\hline \multicolumn{3}{|c|}{ Assessment of outcome (specific disorder) } \\
\hline \multicolumn{3}{|c|}{ Adequate description of the outcome } \\
\hline 7 & Outcome definition & $+/-/ ?$ \\
\hline $8 f$ & Assessment method & $+/-/ ?$ \\
\hline $9 \mathrm{E}$ & Blind for exposure status & $+/-1 ?$ \\
\hline \multicolumn{3}{|c|}{ Study design } \\
\hline 10 & Prospective design & $+/-/ ?$ \\
\hline 11 & Inclusion and exclusion criteria & $+/-/ ?$ \\
\hline 12 & Follow-up period $\geq 1$ year & $+/-/ ?$ \\
\hline 13 & Information between completers versus withdrawals & $+/-/ ?$ \\
\hline \multicolumn{3}{|c|}{ Analysis and data presentation } \\
\hline 14 & Data presentation & $+/-/ ?$ \\
\hline \multicolumn{3}{|c|}{ Identifying confounders } \\
\hline 15 & Consideration of confounders & $+/-/ ?$ \\
\hline 16 & Control for confounding & $+/-/ ?$ \\
\hline
\end{tabular}


Table 2. Studies that report carpal tunnel syndrome (CTS) for one job title in comparison with another job title. ( $A=$ combination of typical symptoms and electrodiagnostic testing, $\mathrm{B}=$ symptoms and physical examination or electrodiagnostic testing, $\mathrm{C}=$ questionnaire only, $\mathrm{CS}$ = cross-sectional, $\mathrm{CC}=$ case-control, $\mathrm{OR}=$ odds ratio, $95 \% \mathrm{Cl}=95 \%$ confidence interval)

\begin{tabular}{|c|c|c|c|c|c|c|}
\hline \multirow[t]{2}{*}{ Author } & \multirow[t]{2}{*}{ Design } & \multirow{2}{*}{$\begin{array}{l}\text { Out- } \\
\text { come- } \\
\text { as- } \\
\text { sess- } \\
\text { ment }\end{array}$} & \multicolumn{2}{|c|}{ Study population } & \multicolumn{2}{|r|}{ Results } \\
\hline & & & Exposed & Reference & $\mathrm{OR}$ & $95 \% \mathrm{Cl}$ \\
\hline $\begin{array}{l}\text { Margolis \& Kraus, } \\
1987(37)\end{array}$ & CS & C & $\begin{array}{l}\text { Female supermarket checkers using laser scan- } \\
\text { ner }(N=691)\end{array}$ & $\begin{array}{l}\text { Female supermarket checkers not using laser } \\
\text { scanner }(\mathrm{N}=291)\end{array}$ & 1.05 & $0.74-1.49$ \\
\hline $\begin{array}{l}\text { Barnhart et al, } \\
1991(33)\end{array}$ & CS & $A$ & Ski manufacturing workers $(\mathrm{N}=106)$ & Repetitive jobs versus nonrepetitive jobs & 1.63 & $0.83-3.21$ \\
\hline $\begin{array}{l}\text { Bovenzi et al, } \\
1991(21)\end{array}$ & CS & $A$ & $\begin{array}{l}\text { Vibration-exposed forestry workers using chain } \\
\text { saws }(N=65)\end{array}$ & $\begin{array}{l}\text { Workers who performed maintenance activities } \\
\text { in a hospital }(\mathrm{N}=31)\end{array}$ & 21.3 & $\begin{array}{c}2.73- \\
166.14\end{array}$ \\
\hline $\begin{array}{l}\text { Moore \& Garg, } \\
1994 \text { (39) }\end{array}$ & CS & $A$ & $\begin{array}{l}\text { Employees with hazardous jobs in a pork-pro- } \\
\text { cessing plant }(\mathrm{N}=79)\end{array}$ & $\begin{array}{l}\text { Employees with safe jobs in a pork-processing } \\
\text { plant ( } N=37)\end{array}$ & 3.34 & $0.92-12.18$ \\
\hline $\begin{array}{l}\text { Frost et al, } 1998 \\
\text { (23) }\end{array}$ & Cohort & $A$ & Slaughterhouse workers ( $\mathrm{N}=743$ ) & $\begin{array}{l}\text { Repairmen or chemical workers in chemical } \\
\text { plant }(\mathrm{N}=398)\end{array}$ & 4.24 & $1.77-10.13$ \\
\hline $\begin{array}{l}\text { Abbas et al, } 2001 \\
\text { (19) }\end{array}$ & CS & A & Electronics assembly workers ( $\mathrm{N}=104)$ & Clerical and administrative workers ( $N=94)$ & 11.4 & $3.6-40.2$ \\
\hline $\begin{array}{l}\text { Kim et al, } 2004 \\
(25)\end{array}$ & CS & A & Meat and fish processing workers $(\mathrm{N}=69)$ & Managers, secretary, and keepers ( $\mathrm{N}=28$ ) & 76.5 & $9.68-604.5$ \\
\hline $\begin{array}{l}\text { Gell et al, } 2005 \\
(35)\end{array}$ & Cohort & A & Industrial workers (N=173) & Clerical workers $(\mathrm{N}=259)$ & 1.06 & $0.49-2.28$ \\
\hline $\begin{array}{l}\text { Bonfiglioli et al, } \\
2007 \text { (34) }\end{array}$ & CS & A & $\begin{array}{l}\text { Cashiers } \\
\text { Part-time }(\mathrm{N}=155) \\
\text { Full-time }(\mathrm{N}=71)\end{array}$ & Office workers ( $\mathrm{N}=98$ ) & $\begin{array}{l}1.06 \\
1.81\end{array}$ & $\begin{array}{l}0.35-3.21 \\
0.52-6.34\end{array}$ \\
\hline $\begin{array}{l}\text { McCormack et al, } \\
1990(38)\end{array}$ & CS & B & $\begin{array}{l}\text { Textile workers } \\
\text { Boarding }(\mathrm{N}=296) \\
\text { Knitting }(\mathrm{N}=352) \\
\text { Packer or folder }(\mathrm{N}=369) \\
\text { Sewing }(\mathrm{N}=562)\end{array}$ & $\begin{array}{l}\text { Cleaners, sweepers, machinery and transport } \\
\text { workers }(\mathrm{N}=468)\end{array}$ & $\begin{array}{l}0.52 \\
0.66 \\
0.42 \\
0.97\end{array}$ & $\begin{array}{l}0.11-2.61 \\
0.16-2.67 \\
0.08-2.09 \\
0.32-2.91\end{array}$ \\
\hline $\begin{array}{l}\text { Park et al, } 1992 \\
\text { (30) }\end{array}$ & CC & B & $\begin{array}{l}\text { Cases with } \geq 1 \text { insurance claims for CTS } \\
\text { Trim or chassis assembly work }(\mathrm{N}=60) \\
\text { Spray painting }(\mathrm{N}=3) \\
\text { Sewing work }(\mathrm{N}=21) \\
\text { Foundry work }(\mathrm{N}=17) \\
\text { Axle assembly work }(\mathrm{N}=9) \\
\text { Stamping and pressing work }(\mathrm{N}=29)\end{array}$ & $\begin{array}{l}\text { Controls with } \geq 1 \text { insurance claims for other } \\
\text { causes }\end{array}$ & $\begin{array}{l}3.1 \\
3.6 \\
2.0 \\
9.0 \\
4.2 \\
2.9\end{array}$ & $\begin{array}{l}2.1-4.6 \\
0.99-13.1 \\
1.1-3.6 \\
4.7-17.1 \\
1.9-9.4 \\
1.8-4.7\end{array}$ \\
\hline $\begin{array}{l}\text { Liss et al, } 1995 \\
\text { (28) }\end{array}$ & CS & C & Dental hygienists $(\mathrm{N}=950)$ & Dental assistants ( $N=108)$ & 3.7 & $1.1-11.9$ \\
\hline $\begin{array}{l}\text { Pocekay et al, } \\
1995(31)\end{array}$ & CS & C & $\begin{array}{l}\text { Photolithography or etching in semiconduc- } \\
\text { tor industry }\end{array}$ & Nonfabrication room & 4.22 & $1.83-9.17$ \\
\hline $\begin{array}{l}\text { Silverstein \& } \\
\text { Hughes, } 1996 \\
(40)\end{array}$ & CS & B & $\begin{array}{l}\text { Workers in power and recovery department in } \\
\text { pulp and paper mill }(\mathrm{N}=23)\end{array}$ & Paper machine operators ( $N=17)$ & 7.0 & $0.77-63.6$ \\
\hline $\begin{array}{l}\text { Leclerc et al, } \\
1998(27)\end{array}$ & CS & B & $\begin{array}{l}\text { Employees in different branches of activity } \\
\text { at the national level (assembly line, clothing } \\
\text { and shoe industry, food industry, supermarket } \\
\text { cashiers) }\end{array}$ & $\begin{array}{l}\text { Jobs with repetitive tasks versus jobs without } \\
\text { repetitive tasks }\end{array}$ & 5.65 & $2.74-11.63$ \\
\hline $\begin{array}{l}\text { Gorsche et al, } \\
1999 \text { (36) }\end{array}$ & CS & B & $\begin{array}{l}\text { Tool users in repetitive jobs in a meat packing } \\
\text { plant }(N=521)\end{array}$ & $\begin{array}{l}\text { Nontool users in repetitive jobs }(\mathrm{N}=89) \text { and } \\
\text { supervisors and clerical workers }(\mathrm{N}=55) \text { in a } \\
\text { meatpacking plant }\end{array}$ & 1.2 & $0.75-1.92$ \\
\hline $\begin{array}{l}\text { Nordander et al, } \\
1999(66)\end{array}$ & CS & B & Fish-processing workers ( $\mathrm{N}=322)$ & $\begin{array}{l}\text { Referents employed as caretakers, workers in } \\
\text { community parks and gardens, workers repair- } \\
\text { ing and maintaining equipment and machines, } \\
\text { day nurses, caretakers }(N=337)\end{array}$ & 5.3 & $0.62-45.61$ \\
\hline Diaz, 2001 (22) & CS & B & Nurse anesthetists ( $\mathrm{N}=63$ ) & Operating room nurses $(\mathrm{N}=181)$ & 3.32 & $1.27-8.17$ \\
\hline $\begin{array}{l}\text { Kutluhan et al, } \\
2001(26)\end{array}$ & CS & B & Female carpet workers ( $\mathrm{N}=140$ hands) & Housewives ( $\mathrm{N}=60$ hands) & 3.98 & $1.34-11.84$ \\
\hline $\begin{array}{l}\text { Jianmongkol et } \\
\text { al, } 2005(24)\end{array}$ & CS & B & Workers in a fishnet factory $(\mathrm{N}=550)$ & Office workers or housemaids ( $\mathrm{N}=112$ ) & 1.84 & $\begin{array}{r}1.03-3.29 \\
\text { (continued) }\end{array}$ \\
\hline
\end{tabular}


Table 2. Continued.

\begin{tabular}{|c|c|c|c|c|c|c|}
\hline \multirow[t]{2}{*}{ Author } & \multirow[t]{2}{*}{ Design } & \multirow{2}{*}{$\begin{array}{l}\text { Out- } \\
\text { come } \\
\text { as- } \\
\text { sess- } \\
\text { ment }\end{array}$} & \multicolumn{2}{|c|}{ Study population } & \multicolumn{2}{|c|}{ Results } \\
\hline & & & Exposed & Reference & $\mathrm{OR}$ & $95 \% \mathrm{Cl}$ \\
\hline $\begin{array}{l}\text { Wang et al, } 2005 \\
\text { (32) }\end{array}$ & CS & B & Female betel pepper leaf cullers $(\mathrm{N}=20)$ & Female noncullers $(\mathrm{N}=47)$ & 9.78 & $2.24-42.63$ \\
\hline $\begin{array}{l}\text { Ali \& } \\
\text { Sathiyasekaran, } \\
2006 \text { (20) }\end{array}$ & CS & B & System administrators $(\mathrm{N}=50)$ & Data entry typists $(\mathrm{N}=244)$ & 2.5 & $1.2-5.2$ \\
\hline $\begin{array}{l}\text { Ali \& } \\
\text { Sathiyasekaran, } \\
2006 \text { (20) }\end{array}$ & CS & B & Software developers $(\mathrm{N}=143)$ & Data entry typists ( $N=244)$ & 1.0 & $0.6-1.9$ \\
\hline
\end{tabular}

Table 3. Studies of associations between physical risk factors (force, repetitiveness, hand-arm vibration, combined exposure measure, posture) and carpal tunnel syndrome (CTS). ( $\mathrm{A}=$ combination of typical symptoms and electrodiagnostic testing, $B=$ symptoms and physical examination or electrodiagnostic testing, $\mathrm{C}=$ questionnaire only, $\mathrm{CS}=$ cross-sectional, $\mathrm{CC}=$ case-control, $\mathrm{OR}=0 \mathrm{dds}$ ratio, $95 \%$ $\mathrm{Cl}=$ confidence interval)

\begin{tabular}{|c|c|c|c|c|c|c|}
\hline \multirow[t]{2}{*}{ Author } & \multirow{2}{*}{$\begin{array}{l}\text { De- } \\
\text { sign }\end{array}$} & \multirow{2}{*}{$\begin{array}{l}\text { Out- } \\
\text { come } \\
\text { as- } \\
\text { sess- } \\
\text { ment }\end{array}$} & \multirow[t]{2}{*}{ Study population } & \multirow[t]{2}{*}{ Physical risk factor } & \multicolumn{2}{|c|}{ Results } \\
\hline & & & & & $\mathrm{OR}$ & $95 \% \mathrm{Cl}$ \\
\hline \multicolumn{7}{|l|}{ Force } \\
\hline $\begin{array}{l}\text { Wieslander et al, } 1989 \\
(46)\end{array}$ & $\mathrm{CC}$ & A & $\begin{array}{l}\text { Men operated upon for CTS ( } 34 \text { cases) versus } \\
\text { other patients with surgery and general popula- } \\
\text { tion referents }(N=143)\end{array}$ & $\begin{array}{l}\text { Work causing great load on wrist } \\
1-20 \text { versus }<1 \text { year } \\
>20 \text { versus }<1 \text { year }\end{array}$ & $\begin{array}{l}1.7 \\
2.1\end{array}$ & $\begin{array}{l}0.7-3.9 \\
0.8-5.5\end{array}$ \\
\hline $\begin{array}{l}\text { de Krom et al, } 1990 \\
\text { (43) }\end{array}$ & $\mathrm{CC}$ & A & $\begin{array}{l}\text { CTS patients in a neurology department of a hos- } \\
\text { pital ( } 156 \text { cases) and a random sample from the } \\
\text { general population ( } 473 \text { referents) }\end{array}$ & $\begin{array}{l}\text { Pinch grasp } \\
1-7 \text { week versus } 0 \text { hours/week } \\
8-19 \text { versus } 0 \text { hours/week } \\
20-40 \text { versus } 0 \text { hours/week }\end{array}$ & $\begin{array}{l}0.9 \\
0.8 \\
0.7\end{array}$ & $\begin{array}{l}0.8-1.1 \\
0.5-1.3 \\
0.3-1.6\end{array}$ \\
\hline $\begin{array}{l}\text { Roquelaure et al, } 1997 \\
\text { (42) }\end{array}$ & $\mathrm{CC}$ & A & $\begin{array}{l}\text { Workers with CTS ( } 65 \text { cases) and workers with- } \\
\text { out CTS ( } 65 \text { referents) in } 3 \text { plants where TV sets, } \\
\text { shoes and automobile brakes were manufactured }\end{array}$ & High force (yes; no) & 9.0 & $2.4-33.4$ \\
\hline $\begin{array}{l}\text { Lam \& Thurston, } 1998 \\
(41)\end{array}$ & $\mathrm{CC}$ & A & $\begin{array}{l}\text { Workers operated upon for CTS ( } 512 \text { cas- } \\
\text { es) and workers in the general population } \\
\text { (1 } 400403 \text { referents) }\end{array}$ & $\begin{array}{l}\text { Manual work } \\
\text { Moderate versus light } \\
\text { Heavy versus light }\end{array}$ & $\begin{array}{l}2.36 \\
2.11\end{array}$ & $\begin{array}{l}1.34-4.13 \\
1.20-3.71\end{array}$ \\
\hline Abbas et al, 2001 (19) & CS & A & Electronics assembly workers $(\mathrm{N}=104)$ & $\begin{array}{l}\text { Precision grip versus power grip } \\
\text { Intermediate grip versus power grip }\end{array}$ & $\begin{array}{l}6.5 \\
2.0\end{array}$ & $\begin{array}{l}1.1-39.2 \\
0.3-11.9\end{array}$ \\
\hline Nathan et al, 2002 (45) & $\begin{array}{l}\text { Co- } \\
\text { hort }\end{array}$ & A & $\begin{array}{l}\text { Industrial workers (steel mill, meat or food } \\
\text { packaging, electronics, plastics) with 11-year fol- }\end{array}$ & $\begin{array}{l}\text { Heavy lifting ( } 1 \text { to } 5 \text { Likert scale) } \\
\text { Force ( } 1 \text { to } 5 \text { Likert scale) }\end{array}$ & $\begin{array}{l}0.99 \\
1.00\end{array}$ & $\begin{array}{l}0.76-1.29 \\
0.75-1.34\end{array}$ \\
\hline Nathan et al, 2005 (44) & $\begin{array}{l}\text { Co- } \\
\text { hort }\end{array}$ & A & $\begin{array}{l}\text { Industrial workers (steel mill, meat or food } \\
\text { packaging, electronics, plastics) with 17-year fol- } \\
\text { low-up (N=148) }\end{array}$ & $\begin{array}{l}\text { Heavy lifting (1 to } 5 \text { Likert scale) } \\
\text { Force (1 to } 5 \text { Likert scale) }\end{array}$ & $\begin{array}{l}0.39^{\mathrm{a}} \\
3.50^{\mathrm{b}}\end{array}$ & $\begin{array}{l}. . \\
. .\end{array}$ \\
\hline \multicolumn{7}{|l|}{ Repetitiveness } \\
\hline $\begin{array}{l}\text { Wieslander et al, } 1989 \\
\text { (46) }\end{array}$ & $\mathrm{CC}$ & A & $\begin{array}{l}\text { Men operated upon for CTS ( } 34 \text { cases) versus } \\
\text { other patients with surgery and other referents } \\
\text { from the general population }(N=143)\end{array}$ & $\begin{array}{l}\text { Repetitive movement of wrist } \\
1-20 \text { versus }<1 \text { year } \\
>20 \text { versus }<1 \text { year }\end{array}$ & $\begin{array}{l}1.5 \\
4.6\end{array}$ & $\begin{array}{c}0.5-4.4 \\
1.8-11.9\end{array}$ \\
\hline Chiang et al, 1990 (47) & CS & A & Frozen food factory employees $(\mathrm{N}=207)$ & $\begin{array}{l}\text { High repetitive movements ver- } \\
\text { sus no cold and low repetitive } \\
\text { movements } \\
\text { Handling cold items }\left(-12-15^{\circ} \mathrm{C}\right) \\
\text { and high repetitive movements } \\
\text { versus no cold and low repetitive } \\
\text { movements }\end{array}$ & 2.18 & $0.23-21.27$ \\
\hline $\begin{array}{l}\text { Roquelaure et al, } 1997 \\
\text { (42) }\end{array}$ & $\mathrm{CC}$ & A & $\begin{array}{l}\text { Workers with CTS ( } 65 \text { cases) and workers with- } \\
\text { out CTS ( } 65 \text { referents) in } 3 \text { plants where TV sets, } \\
\text { shoes and automobile brakes were manufactured }\end{array}$ & Work cycle <10 seconds (yes; no) & 8.8 & $1.8-44.4$ \\
\hline
\end{tabular}


Table 3. Continued.

\begin{tabular}{|c|c|c|c|c|c|c|}
\hline \multirow[t]{2}{*}{ Author } & \multirow{2}{*}{$\begin{array}{l}\text { De- } \\
\text { sign }\end{array}$} & \multirow{2}{*}{$\begin{array}{l}\text { Out- } \\
\text { come } \\
\text { as- } \\
\text { sess- } \\
\text { ment }\end{array}$} & \multirow[t]{2}{*}{ Study population } & \multirow[t]{2}{*}{ Physical risk factor } & \multicolumn{2}{|c|}{ Results } \\
\hline & & & & & $\mathrm{OR}$ & $95 \% \mathrm{Cl}$ \\
\hline Latko et al, 1999 (50) & CS & A & Manufacturing workers ( $\mathrm{N}=352$ ) & $\begin{array}{l}\text { Medium repetitive work versus low } \\
\text { repetitive work } \\
\text { High repetitive work versus low re- } \\
\text { petitive work }\end{array}$ & $\begin{array}{l}1.95 \\
3.13\end{array}$ & $\begin{array}{l}0.38-9.96 \\
0.87-11.25\end{array}$ \\
\hline Nathan et al, 2002 (45) & $\begin{array}{l}\text { Co- } \\
\text { hort }\end{array}$ & A & $\begin{array}{l}\text { Industrial workers (steel mill, meat or food pack- } \\
\text { aging, electronics, plastics) with 11-year follow- } \\
\text { up ( } N=471)\end{array}$ & Repetition (1 to 5 Likert scale) & 1.05 & $0.79-1.39$ \\
\hline Nathan et al, 2005 (44) & $\begin{array}{l}\text { Co- } \\
\text { hort }\end{array}$ & A & $\begin{array}{l}\text { Industrial workers (steel mill, meat or food pack- } \\
\text { aging, electronics, plastics) with 17-year follow- } \\
\text { up (N=166) }\end{array}$ & Repetition (1 to 5 Likert scale) & $0.50^{c}$ & .. \\
\hline $\begin{array}{l}\text { Roquelaure et al, } 2001 \\
\text { (49) }\end{array}$ & CS & B & $\begin{array}{l}\text { Workers }(\mathrm{N}=134) \text { in } 5 \text { production units of a large, } \\
\text { modern mechanized footwear factory }\end{array}$ & $\begin{array}{l}\text { Work cycle time <30 seconds (yes; } \\
\text { no) } \\
\text { Rapid trigger movements (yes; no) }\end{array}$ & $\begin{array}{l}0.7 \\
2.8\end{array}$ & $\begin{array}{c}0.2-2.3 \\
0.6-11.5\end{array}$ \\
\hline $\begin{array}{l}\text { Babski-Reeves \& } \\
\text { Crumtpon-Young, } \\
2002 \text { (48) }\end{array}$ & CS & B & Employees of a fish-processing facility $(\mathrm{N}=53)$ & $\begin{array}{l}\text { High repetition (cycle time }<30 \text { sec- } \\
\text { onds) versus low repetition }\end{array}$ & 0.96 & $0.35-2.64$ \\
\hline \multicolumn{7}{|l|}{ Hand-arm vibration } \\
\hline $\begin{array}{l}\text { Wieslander et al, } 1989 \\
\text { (46) }\end{array}$ & CC & A & $\begin{array}{l}\text { Men operated upon for CTS ( } 34 \text { cases) versus } \\
\text { other patients with surgery and general popula- } \\
\text { tions referents }(N=143)\end{array}$ & $\begin{array}{l}\text { Use of vibrating tools } \\
1-20 \text { versus }<1 \text { year } \\
>20 \text { versus }<1 \text { year }\end{array}$ & $\begin{array}{l}2.7 \\
4.8\end{array}$ & $\begin{array}{l}1.1-6.7 \\
1.5-15.6\end{array}$ \\
\hline Nathan et al, 2002 (45) & $\begin{array}{l}\text { Co- } \\
\text { hort }\end{array}$ & A & $\begin{array}{l}\text { Industrial workers (steel mill, meat or food pack- } \\
\text { aging, electronics, plastics) with 11-year follow- } \\
\text { up ( } N=471)\end{array}$ & Vibrations (yes; no) & 2.28 & $0.84-6.29$ \\
\hline $\begin{array}{l}\text { Nordstrom et al, } 1997 \\
\text { (52) }\end{array}$ & $\mathrm{CC}$ & B & $\begin{array}{l}\text { Workers with newly diagnosed CTS ( } 182 \text { cases ) } \\
\text { and random sample of workers from the general } \\
\text { population ( } 188 \text { referents) }\end{array}$ & $\begin{array}{l}\text { Power tools or machinery (mean) } \\
0.08-0.75 \text { versus } 0 \text { hours/day } \\
1-2 \text { versus } 0 \text { hours/day } \\
2.5-5.5 \text { versus } 0 \text { hours/day } \\
6-11 \text { versus } 0 \text { hours/day }\end{array}$ & $\begin{array}{l}0.60 \\
1.43 \\
1.20 \\
2.52\end{array}$ & $\begin{array}{l}0.27-1.36 \\
0.66-3.13 \\
0.59-2.45 \\
1.13-5.62\end{array}$ \\
\hline $\begin{array}{l}\text { Roquelaure et al, } 2001 \\
\text { (49) }\end{array}$ & CS & B & $\begin{array}{l}\text { Workers ( } N=134) \text { in } 5 \text { production units of a large, } \\
\text { modern mechanized footwear factory }\end{array}$ & $\begin{array}{l}\text { Vibration transmitted to the hand } \\
\text { (yes; no) }\end{array}$ & 1.8 & $0.2-10.8$ \\
\hline $\begin{array}{l}\text { Bovenzi et al, } 2005 \\
\text { (51) }\end{array}$ & CS & B & $\begin{array}{l}\text { Female furniture workers }(N=100) \text { and female of- } \\
\text { fice workers of the NHS }(N=100)\end{array}$ & $\begin{array}{l}\text { Vibration }\left(4.7 \mathrm{~m} / \mathrm{s}^{2}\right)+\text { physical } \\
\text { stress factors versus no vibration or } \\
\text { physical stress factors } \\
\text { Vibration }\left(3.9 \mathrm{~m} / \mathrm{s}^{2}\right)+\text { physical } \\
\text { stress factors versus no vibration or } \\
\text { physical stress factors } \\
\text { Physical stress factors alone ver- } \\
\text { sus no vibration or physical stress } \\
\text { factors }\end{array}$ & 3.1 & $\begin{array}{l}1.0-13.7 \\
1.3-7.2 \\
0.2-12.0\end{array}$ \\
\hline \multicolumn{7}{|c|}{ Combined exposure measure } \\
\hline Chiang et al, 1993 (53) & CS & A & Workers in the fish-processing industry $(\mathrm{N}=207)$ & $\begin{array}{l}\text { High force or high repetition versus } \\
\text { low force and low repetition } \\
\text { High force and high repetitive versus } \\
\text { low force and low repetition }\end{array}$ & $\begin{array}{l}2.02 \\
4.48\end{array}$ & $\begin{array}{l}0.71-5.72 \\
1.31-15.3\end{array}$ \\
\hline $\begin{array}{l}\text { Silverstein et al, } 1987 \\
\text { (54) }\end{array}$ & CS & B & $\begin{array}{l}\text { Employees from } 7 \text { different industrial sites } \\
(\mathrm{N}=652)\end{array}$ & $\begin{array}{l}\text { High force and low repetition versus } \\
\text { low force and low repetition } \\
\text { Low force and high repetition versus } \\
\text { low force and low repetition } \\
\text { High force and high repetition versus } \\
\text { low force-low repetition }\end{array}$ & $\begin{array}{l}1.62 \\
3.34 \\
8.38\end{array}$ & $\begin{array}{l}0.15-17.99 \\
0.34-32.51 \\
1.03-67.78\end{array}$ \\
\hline Yagev et al, 2001 (55) & CC & B & $\begin{array}{l}\text { Workers with CTS diagnosed in the hospital ( } 123 \\
\text { cases) and workers without CTS but referred for } \\
\text { complaints ( } 246 \text { referents) }\end{array}$ & $\begin{array}{l}\text { Low force and high repetitive job } \\
\text { versus low force and low repeti- } \\
\text { tive job } \\
\text { High force and low repetitive job ver- } \\
\text { sus low force and low repetitive job }\end{array}$ & 4.72 & $1.8-12.5$ \\
\hline $\begin{array}{l}\text { Cosgrove et al, } 2002 \\
(56)\end{array}$ & CC & B & $\begin{array}{l}\text { Railroad workers with confirmed CTS in a physi- } \\
\text { cal examination ( } 389 \text { cases) and railroad workers } \\
\text { with CTS complaints but without a confirmed } \\
\text { diagnosis ( } 511 \text { referents) from the compensation } \\
\text { claim system }\end{array}$ & $\begin{array}{l}\text { Low force and high repetition versus } \\
\text { low force and low repetition } \\
\text { High force and low repetition versus } \\
\text { low force and low repetition } \\
\text { High force and high repetition versus } \\
\text { low force and low repetition }\end{array}$ & $\begin{array}{l}0.72 \\
0.84 \\
1.09\end{array}$ & $\begin{array}{l}0.47-1.09 \\
0.59-1.18 \\
0.21-5.54\end{array}$ \\
\hline
\end{tabular}


Table 3. Continued.

\begin{tabular}{|c|c|c|c|c|c|c|}
\hline \multirow[t]{2}{*}{ Author } & \multirow{2}{*}{$\begin{array}{l}\text { De- } \\
\text { sign }\end{array}$} & \multirow{2}{*}{$\begin{array}{l}\text { Out- } \\
\text { come } \\
\text { as- } \\
\text { sess- } \\
\text { ment }\end{array}$} & \multirow[t]{2}{*}{ Study population } & \multirow[t]{2}{*}{ Physical risk factor } & \multicolumn{2}{|c|}{ Results } \\
\hline & & & & & OR & $95 \% \mathrm{Cl}$ \\
\hline
\end{tabular}

\section{Posture}

de Krom et al, 1990

(43)

$\begin{array}{llll}\text { Lam \& Thurston, 1998 } & \text { CC } & \text { A } & \begin{array}{l}\text { Workers operated upon for CTS (512 cas- } \\ \text { es) and workers in general population } \\ (141)\end{array} \\ \text { Stevens et al, } 2001 & \text { CS } & \text { A } & \begin{array}{l}(1400403 \text { referents }) \\ \text { Computer users at a medical facility }(\mathrm{N}=249)\end{array}\end{array}$
CC pital (156 cases) and a random sample from the general population (473 referents)
Nathan et al, 2002 (45) Cohort

Morgenstern et al, 1991 (29)

Blanc et al, 1996 (58)

Nordstrom et al, 1997 (52)

Roquelaure et al, 2001 (49)

Andersen et al, 2003 (57)

CS
A Industrial workers (steel mill, meat or food packaging, electronics, plastics) with 11-year followup $(\mathrm{N}=471)$

CS

C Female grocery checkers $(\mathrm{N}=1053)$

Activities with flexed wrist $0-5$ years

ago

$1-7$ versus 0 hours/week 8-19 versus 0 hours/week

20-40 versus 0 hours/week

Activities with extended wrist

$1-7$ versus 0 hours/week

8-19 versus 0 hours/week

20-40 versus 0 hours/week

Activities with extended and flexed

wrist in combination

$1-7$ versus 0 hours/week

$8-19$ versus 0 hours/week

20-40 versus 0 hours/week

Typing

$1-7$ versus 0 hours/week

8-19 versus 0 hours/week

20-40 versus 0 hours/week

Clerical work (long keyboard use

versus short keyboard use)

$1.3-1.9$

$1.8-4.9$

3.1-24.1

$1.0-1.9$

$1.0-5.2$

$1.1-27.4$

$0.9-1.2$

$0.8-1.7$

$0.7-2.9$

$0.6-1.4$

$0.3-2.5$

$0.1-6.0$

1.01

$0.52-1.95$

Occasionally mouse use versus no mouse use

$0.32-7.04$

Frequently mouse use versus no

mouse use

Currently typewriter use versus no

typewriter use

$0.71-15.8$

Keyboard use (1 to 5 Likert scale)

$0.40-2.03$

0.90

$0.74-1.23$

Workhours per week

26-34 versus $<26$ hours

$>34$ versus $<26$ hours

Years worked

$5-9$ versus $<5$ years

$>9$ versus $<5$ years

Hand-bending more than

2 hours/day (yes; no) (27 260 referents)

Bending hands or wrists (mean)

$0.25-1.75$ versus 0 hours/day

$2-3$ versus 0 hours/day

$3.5-6$ versus 0 hours/day

$7-16$ versus 0 hours/day

B

Workers $(\mathrm{N}=134)$ in 5 production units of a large, modern mechanized footwear factory

Wrist deviation (yes/no)

Wrist flexion >45 degrees (yes; no)

Wrist extension $>45$ degrees (yes:

no)

$1.53 \quad 0.95-2.44$

$1.86 \quad 1.12-3.08$

$0.91 \quad 0.53-1.58$

$1.74 \quad 0.96-3.16$

1.3

$1.2-1.4$

$0.64-2.80$

$0.60-2.53$

$1.24-4.36$

$1.38-4.43$

$0.4-3.1$

$0.4-3.4$

Right-handed mouse use

\section{$2.5-<5$ versus $0-<2.5$ hours/week $0.7 \quad 0.3-1.9$}

$5-<10$ versus $0-<2.5$ hours/week $\quad 1.9 \quad 0.9-4.0$

$10-<15$ versus $0-<2.5$ hours/week $\quad 1.6 \quad 0.8-3.3$

$15-<20$ versus $0-<2.5$ hours/week $\quad 2.0 \quad 0.9-4.2$

$20-<25$ versus $0-<2.5$ hours/week $2.6 \quad 1.2-5.5$

$25-<30$ versus $0-<2.5$ hours/week $3.2 \quad 1.3-7.9$

$\geq 30$ versus $0-<2.5$ hours/week $\quad 2.7 \quad 1.0-7.6$

Keyboard use

$2.5<5$ versus $0-<2.5$ hours/week $\quad 0.9 \quad 0.4-1.8$

$5<10$ versus $0-<2.5$ hours/week $\quad 0.8 \quad 0.4-1.5$

$10<15$ versus $0-<2.5$ hours/week $\quad 1.2 \quad 0.6-2.5$

$5<20$ versus $0-<2.5$ hours/week $\quad 0.8 \quad 0.4-1.5$

$\geq 20$ versus $-<2.5$ hours/week $\quad 1.4 \quad 0.5-4.3$ 
Table 3. Continued.

\begin{tabular}{|c|c|c|c|c|c|c|}
\hline \multirow[t]{2}{*}{ Author } & \multirow{2}{*}{$\begin{array}{l}\text { De- } \\
\text { sign }\end{array}$} & \multirow{2}{*}{$\begin{array}{l}\text { Out- } \\
\text { come } \\
\text { as- } \\
\text { sess- } \\
\text { ment }\end{array}$} & \multirow{2}{*}{ Study population } & \multirow[t]{2}{*}{ Physical risk factor } & \multicolumn{2}{|c|}{ Results } \\
\hline & & & & & $\mathrm{OR}$ & $95 \% \mathrm{Cl}$ \\
\hline $\begin{array}{l}\text { Ali \& Sathiyasekaran, } \\
2006 \text { (20) }\end{array}$ & CS & $\mathrm{B}$ & Computer professionals ( $\mathrm{N}=648$ ) & $\begin{array}{l}\text { Hand position } \\
\text { Flexed or extended versus neutral } \\
\text { Computer work } \\
8-1 \text { versus }<8 \text { hours/day } \\
>12 \text { versus }<8 \text { hours/day } \\
4-8 \text { versus }<4 \text { years } \\
>8 \text { versus }<4 \text { years }\end{array}$ & $\begin{array}{l}1.3 \\
3.6 \\
4.4 \\
2.1 \\
2.7\end{array}$ & $\begin{array}{c}0.8-2.1 \\
1.3-10.3 \\
1.3-14.9 \\
1.3-3.6 \\
1.3-5.8\end{array}$ \\
\hline Hou et al, 2007 (59) & CS & C & Male operators of visual display terminal $(\mathrm{N}=340)$ & $\begin{array}{l}\text { Daily keyboard operation hours } \\
\text { ( } \geq 4 \text { versus }<4 \text { hours/day) } \\
\text { Daily mouse operation hours (h/day) } \\
\text { ( } \geq 4 \text { versus }<4 \text { hours/day) }\end{array}$ & $\begin{array}{l}1.34 \\
0.85\end{array}$ & $\begin{array}{l}0.36-5.00 \\
0.27-2.65\end{array}$ \\
\hline
\end{tabular}

a P-value 0.070 .

b P-value 0.064 .

c P-value 0.046 .

Table 4. Studies on associations between psychosocial risk factors and carpal tunnel syndrome (CTS). ( $A=$ combination of typical symptoms and electrodiagnostic testing, $\mathrm{B}=$ symptoms and physical examination or electrodiagnostic testing, CS = cross-sectional, CC = case-control, $\mathrm{OR}=$ odds ratio, $95 \% \mathrm{Cl}=95 \%$ confidence interval)

\begin{tabular}{|c|c|c|c|c|c|c|}
\hline \multirow[t]{2}{*}{ Author } & \multirow[t]{2}{*}{ Design } & \multirow{2}{*}{$\begin{array}{l}\text { Outcome } \\
\text { assess- } \\
\text { ment }\end{array}$} & \multirow[t]{2}{*}{ Study population } & \multirow[t]{2}{*}{ Psychosocial risk factor } & \multicolumn{2}{|c|}{ Results } \\
\hline & & & & & $\mathrm{OR}$ & $95 \% \mathrm{Cl}$ \\
\hline $\begin{array}{l}\text { Werner et al, } \\
2005(67)\end{array}$ & Cohort & $A$ & $\begin{array}{l}\text { Auto assembly workers }(\mathrm{N}=166) \text { with a 1-year } \\
\text { follow-up }\end{array}$ & $\begin{array}{l}\text { High social support versus low social } \\
\text { support }\end{array}$ & 0.69 & $0.48-0.99$ \\
\hline $\begin{array}{l}\text { Nordstrom et al, } \\
1997(52)\end{array}$ & CC & $B$ & $\begin{array}{l}\text { Workers with newly diagnosed CTS (182 cas- } \\
\text { es) and a random sample of workers from the } \\
\text { general population (188 referents) }\end{array}$ & $\begin{array}{l}\text { Job control ( } 1 \text { very little to } 5 \text { very much) } \\
2.8-3.4 \text { versus } 1-2.7 \\
3.6-3.8 \text { versus } 1-2.7 \\
4-4.4 \text { versus } 1-2.7 \\
4.6-4.8 \text { versus } 1-2.7\end{array}$ & $\begin{array}{l}0.80 \\
0.36 \\
0.46 \\
0.42\end{array}$ & $\begin{array}{l}0.44-1.47 \\
0.18-0.71 \\
0.24-0.86 \\
0.21-0.83\end{array}$ \\
\hline $\begin{array}{l}\text { Roquelaure et al, } \\
2001 \text { (49) }\end{array}$ & CS & $\mathrm{B}$ & $\begin{array}{l}\text { Workers }(\mathrm{N}=134) \text { in } 5 \text { production units of a } \\
\text { large, modern mechanized footwear factory }\end{array}$ & $\begin{array}{l}\text { Permanent time pressure (yes; no) } \\
\text { Few possibilities to take breaks (yes; no) } \\
\text { Work strongly controlled by superiors } \\
\text { (yes; no) } \\
\text { High score for work demand (yes; no) } \\
\text { Low score for task control (yes; no) } \\
\text { Low score for social support (yes; no) }\end{array}$ & $\begin{array}{l}1.3 \\
2.5 \\
0.5 \\
1.7 \\
1.3 \\
1.6\end{array}$ & $\begin{array}{l}0.4-4.1 \\
0.8-8.1 \\
0.2-1.3 \\
0.5-5.4 \\
0.4-3.8 \\
0.5-4.9\end{array}$ \\
\hline $\begin{array}{l}\text { Andersen et al, } \\
2003(57)\end{array}$ & Cohort & $\mathrm{B}$ & $\begin{array}{l}\text { Professional technicians }(\mathrm{N}=5658) \text { with a 1- } \\
\text { year follow-up }\end{array}$ & $\begin{array}{l}\text { High demands (yes; no) } \\
\text { Low control (yes; no) } \\
\text { Low social support (yes; no) } \\
\text { Time pressure (yes; no) }\end{array}$ & $\begin{array}{l}1.3 \\
0.9 \\
1.2 \\
1.0\end{array}$ & $\begin{array}{l}0.9-1.8 \\
0.7-1.4 \\
0.9-1.8 \\
0.7-1.6\end{array}$ \\
\hline
\end{tabular}

Studies reporting CTS by job title (table 2) in comparison with articles that reported the risk of CTS by physical and psychosocial risk factors (tables 3 and 4) had a lower mean quality score, although not significant, 8.05 versus 9.23 , respectively. Figure 2 shows that the methodological quality score of the included articles did not improve over time. Furthermore, the methodological quality score is not related to the level of significance of the association between occupation or risk factors and the occurrence of CTS.

\section{Outcome assessment}

In tables 2-4, the included studies have been classified by the method of outcome assessment. In
19 studies (43\%), the diagnosis of CTS included both the presence of typical symptoms (numbness, tingling, burning, or pain in median innervate fingers) and a positive electrodiagnostic finding (impairment of median nerve function at the wrist). In the rest of the articles, the diagnosis of CTS included symptoms $(\mathrm{N}=9,20 \%)$, a physical examination $(\mathrm{N}=2,5 \%)$, an electrodiagnostic examination $(\mathrm{N}=2,5 \%)$, or a combination of symptoms and a physical examination $(\mathrm{N}=12,27 \%)$. Of the studies with an accurate diagnostic method (ie, typical symptoms and electrodiagnostic examination), 58\% $(\mathrm{N}=11)$ reported a significant association between work-related factors and CTS, against $64 \%(\mathrm{~N}=16)$ of the studies with a less accurate diagnostic method. 
Table 5. Methodological quality scores of the included articles.

\begin{tabular}{|c|c|c|c|c|c|c|c|c|c|c|c|c|c|c|c|c|c|}
\hline Reference & 1 & 2 & 3 & 4 & 5 & 6 & 7 & 8 & 9 & 10 & 11 & 12 & 13 & 14 & 15 & 16 & Score \\
\hline Andersen et al, 2003 (57) & + & + & + & + & + & + & + & + & + & + & $?$ & + & + & + & + & + & 15 \\
\hline Frost et al, 1998 (23) & + & + & + & + & + & + & + & + & - & - & + & + & + & + & + & + & 14 \\
\hline Silverstein et al, 1987 (54) & + & + & - & + & + & + & + & + & + & - & + & - & + & + & + & + & 13 \\
\hline Roquelaure et al, 2001 (49) & + & + & - & + & + & $?$ & + & + & $?$ & + & + & + & + & + & + & + & 13 \\
\hline Barnhart et al, 1991 (33) & + & - & - & + & + & + & + & + & + & - & + & - & + & + & + & + & 12 \\
\hline Gorsche et al, 1999 (36) & + & + & - & + & - & - & + & + & - & + & + & + & + & + & + & + & 12 \\
\hline Latko et al, 1999 (50) & + & + & - & + & + & + & + & + & + & - & + & - & - & + & + & + & 12 \\
\hline Gell et al, 2005 (35) & + & + & - & + & - & + & + & $?$ & $?$ & + & + & + & + & + & + & + & 12 \\
\hline Bovenzi et al, 2005 (51) & + & $?$ & - & + & + & + & + & + & + & - & + & - & - & + & + & + & 11 \\
\hline Leclerc et al, 1998 (27) & + & + & + & + & + & - & + & + & - & - & + & - & - & + & + & + & 11 \\
\hline Nathan et al, 2002 (45) & + & - & - & + & - & + & + & + & $?$ & + & + & + & - & + & + & + & 11 \\
\hline Werner et al, 2005 (67) & + & - & - & + & + & $?$ & + & + & $?$ & + & + & + & - & + & + & + & 11 \\
\hline Wieslander et al, 1989 (46) & - & + & - & + & - & - & + & + & + & - & + & - & + & + & + & - & 10 \\
\hline Chiang et al, 1990 (47) & + & $?$ & + & + & $?$ & + & + & + & + & - & - & - & - & + & + & + & 10 \\
\hline Chiang et al, 1993 (53) & + & $?$ & - & + & + & + & + & + & $?$ & - & + & - & - & + & + & + & 10 \\
\hline Roquelaure et al, 1997 (42) & + & $?$ & + & + & + & + & + & + & + & - & + & - & - & + & - & - & 10 \\
\hline Bovenzi et al, 1991 (21) & - & + & - & + & + & - & + & + & $?$ & - & + & - & + & + & + & + & 10 \\
\hline Nathan et al, 2005 (44) & - & - & - & + & - & + & + & + & $?$ & + & - & + & + & + & + & + & 10 \\
\hline Ali \& Sathiyasekaran, 2006 (20) & - & + & + & + & - & - & + & + & - & - & + & - & + & + & + & + & 10 \\
\hline Nordstrom et al, 1997 (52) & + & + & + & + & - & - & + & - & $?$ & - & + & - & - & + & + & + & 9 \\
\hline Nordander et al, 1999 (66) & - & + & + & + & + & + & + & + & - & - & + & - & - & + & - & - & 9 \\
\hline Jianmongkol et al, 2005 (24) & + & + & + & - & + & $?$ & + & + & $?$ & - & + & - & + & + & - & - & 9 \\
\hline Bonfiglioli et al, 2007 (34) & - & + & - & + & + & $?$ & + & + & - & - & + & - & - & + & + & + & 9 \\
\hline de Krom et al, 1990 (43) & - & + & + & $?$ & - & - & + & + & $?$ & - & + & - & $?$ & + & + & + & 8 \\
\hline Liss et al, 1995 (28) & + & - & + & + & - & - & + & - & - & - & + & - & - & + & + & + & 8 \\
\hline Abbas et al, 2001 (19) & + & + & - & - & - & $?$ & + & + & $?$ & - & - & - & + & + & + & + & 8 \\
\hline Kim et al, 2004 (25) & + & + & + & + & - & - & + & + & $?$ & - & + & - & $?$ & + & - & - & 8 \\
\hline McCormack et al, 1990 (38) & + & + & - & + & - & $?$ & + & + & $?$ & + & - & - & - & + & - & - & 7 \\
\hline Morgenstern et al, 1991 (29) & - & + & + & - & + & $?$ & + & - & - & - & - & - & - & + & + & + & 7 \\
\hline Moore, \& Garg, 1994 (39) & - & $?$ & - & + & + & + & + & - & + & - & + & $?$ & $?$ & + & - & - & 7 \\
\hline Stevens et al, 2001 (60) & + & + & - & - & - & - & + & + & $?$ & - & + & - & + & + & - & - & 7 \\
\hline Yagev et al, 2001 (55) & - & $?$ & + & + & - & - & + & + & - & - & - & - & - & + & + & + & 7 \\
\hline Cosgrove et al, 2002 (56) & $?$ & $?$ & + & + & $?$ & $?$ & + & + & $?$ & - & + & - & $?$ & $?$ & + & + & 7 \\
\hline Park et al, 1992 (30) & - & $?$ & + & - & - & $?$ & + & - & $?$ & - & + & - & - & + & + & + & 6 \\
\hline Blanc et al, 1996 (58) & - & $?$ & + & + & + & - & + & - & - & - & + & - & - & + & - & - & 6 \\
\hline Diaz, 2001 (22) & + & $?$ & - & $?$ & + & $?$ & + & + & - & - & + & - & - & + & - & - & 6 \\
\hline Hou et al, 2007 (59) & + & - & - & + & $?$ & - & - & - & - & - & + & - & - & + & + & + & 6 \\
\hline Margolis \& Kraus, 1987 (37) & - & + & + & - & + & - & - & - & - & - & + & - & - & + & - & - & 5 \\
\hline Pocekay et al, 1995 (31) & - & + & + & + & - & - & + & - & - & - & - & - & - & + & - & - & 5 \\
\hline Silverstein \& Hughes, 1996 (40) & - & - & - & + & - & $?$ & + & + & $?$ & - & + & - & - & - & - & - & 4 \\
\hline Lam \& Thurston, 1998 (41) & - & - & + & + & - & - & + & - & $?$ & - & - & - & - & + & - & - & 4 \\
\hline Kutluhan et al, 2001 (26) & - & $?$ & - & - & + & $?$ & + & + & $?$ & - & $?$ & - & $?$ & + & - & - & 4 \\
\hline Babski-Reeves \& Crumtpon-Young, 2002 (48) & - & $?$ & - & + & + & $?$ & + & - & + & - & - & - & - & - & - & - & 4 \\
\hline Wang et al, 2005 (32) & - & - & - & - & - & $?$ & + & + & $?$ & - & + & - & - & + & - & - & 4 \\
\hline
\end{tabular}

\section{Job title and carpal tunnel syndrome}

A total of 14 articles described significant differences in the occurrence of CTS between occupations, the OR values ranging from 1.86 to 76.5 (19-32). In eight studies, no significant differences were observed, the OR values ranging from 0.91 to 1.63 (33-40). Jobs with the highest increased occurrence of CTS included work in the meatand fish-processing industry (OR 76.5), forestry work with chain saws (OR 21.3), and electronic assembly work (OR 11.4). Other jobs with an elevated occurrence of CTS (OR 2.0-5.0) were work in slaughterhouses, carpet work, and dental work. In these occupations the prevalence of CTS ranged from $6.3 \%$ to $22.8 \%$.

\section{Exposure and carpal tunnel syndrome}

Force. Three articles found a significant association between exposure to force and CTS, with OR values of 2.11 to $9.0(19,41,42)$. In the case-control study of Roquelaure et al (42), handling loads of $>1 \mathrm{~kg}$ at least 10 times/hour was a risk factor for CTS. Moderate and heavy manual work, as reported by Lam et al (41), and 


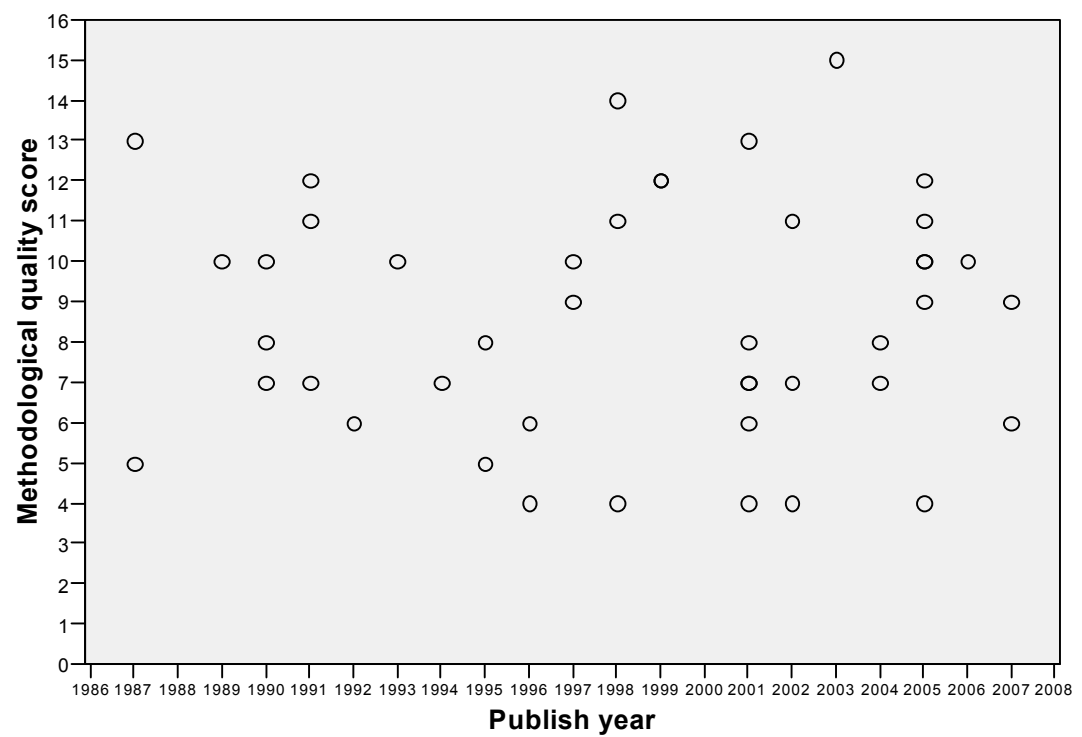

Figure 2. Association between methodological quality and the year of publication for all of the articles that evaluated the association between job title or risk factors and the development of carpal tunnel syndrome. precision grip, as reported by Abbas et al (19), were associated with CTS, but these factors were not described in more detail. Null associations were reported in four articles, in which the duration of work with high loads on the wrist, the duration of using a pinch grasp, heavy lifting, and the relative intensity of force were described, the OR values ranging from 0.39 to 3.50 (43-46).

Repetitiveness. Five articles found a significant association between exposure to repetition and CTS, the OR values ranging from 0.50 to 9.39 (42, 44-47). The strongest associations were found for jobs with a work cycle of $<10$ seconds $(42,47)$. Contradictory results were observed for jobs with work cycles of $<30$ seconds, one study reporting an elevated OR value of 2.18 and other studies reporting OR values close to unity, with 0.7 and 0.96 , respectively (47-49). Five articles reported null associations between exposure to repetitiveness and CTS (46-50).

Hand-arm vibration. Three articles reported a significant association between exposure to vibration and CTS, with OR values of 2.52 to $4.8(46,51,52)$. An increased risk of CTS was found for workers using vibrating tools (120 years and $>20$ years) and power tools or machinery (6-11 hours/day) $(46,52)$. In the cross-sectional study of Bovenzi et al (51), 8-hour time-weighted exposures of $4.7 \mathrm{~m} / \mathrm{s}^{2}$ and $3.9 \mathrm{~m} / \mathrm{s}^{2}$ to hand-arm vibration were associated with CTS. Positive associations between hand-arm vibration and CTS, although not significant, were described by Roquelaure et al (49) and Nathan et al (45), with OR values of 1.8 and 2.28, respectively. The reported prevalence of CTS in both studies was around $14 \%$ with a prevalence of exposure to hand-arm vibration of $17 \%$.
Combined exposure measure. Three cross-sectional studies found a significant relationship between exposure to a combination of risk factors (force, repetitiveness) and CTS (53-55). Silverstein et al (54) and Chiang et al (53) reported a significant relationship between exposure to high force-high repetition and CTS, with OR values of 8.38 and 4.48, respectively. In a study by Yagev et al (55) significant associations were also found between exposure to low force-high repetition (OR 4.72) and high force-low repetition (OR 3.21) and CTS, but these associations were not confirmed in other studies. The case-control study of Cosgrove et al (56) did not corroborate any of these findings.

Posture. Six articles presented significant associations between postural load and CTS $(20,29,43,52,57,58)$. Significant associations were found for activities with a flexed wrist for 1-7, 8-19 and 20-40 hours/week, activities with an extended wrist for 1-7, 8-19 and 20-40 hours/week, and bending hands or wrists for $3.5-6$ and $7-16$ hours/day, the OR values ranging from 1.3 to $8.7(43,52,58)$. In contrast are the results of Roquelaure et al (49) and Ali et al (20), who reported null associations between CTS and wrist deviation, wrist flexion, and wrist extension, the OR values ranging from 0.6 to 1.3 .

In two studies, self-reported exposure to computer work for $>8$ hours/day and mouse use for $>20$ hours/week were associated with CTS, with OR values of 3.6 and 2.6 , respectively $(20,57)$. In contrast, in five studies, no significant associations were found between CTS and the duration of keyboard use, the duration of mouse use, or the frequency of mouse use (OR 0.9-3.4) (41, $45,57,59,60)$. 


\section{Psychosocial risk factors and carpal tunnel syndrome}

Table 4 presents the characteristics of four studies that reported associations between psychosocial risk factors and CTS. None of the reported associations were statistically significant.

\section{Discussion}

This review evaluated the associations between exposure to physical and psychosocial risk factors and CTS. Frequent handling of loads, highly repetitive work with and without force requirements, hand-arm vibration, and activities with a flexed or extended wrist were associated with CTS. Occupations with the highest prevalence of CTS were jobs in the meat- and fish-processing industry, forestry work with chain saws, and electronic assembly work. Contradictory findings were observed for the duration of computer work.

The aforementioned conclusions are primarily based on evidence presented in cross-sectional and case-control studies, since only a small number of cohort studies was identified. Therefore, the causality of the reported associations between exposure and the occurrence of CTS cannot be established, especially since most of the studies relied on self-reported measures of exposure. The lack of cohort studies can partly be explained by the relatively modest incidence of CTS in occupational populations at risk. Nathan and his colleagues found 34 incident CTS cases among 256 industrial workers during an 11-year follow-up, resulting in an estimated incidence of 12.0 per 1000 person-years (45). In the prospective study of Gell et al (35) among 432 industrial and clerical workers with an average follow-up of 5.4 years, a similar incidence of 12.4 per 1000 person-years was observed. Given this low incidence, cohort studies with a low exposure prevalence will be strongly underpowered with respect to demonstrating significant associations, even with OR values of 2.0 or higher, unless very large populations are studied. The lack of sufficient power in studies is also demonstrated by the large confidence intervals in many cross-sectional studies, whereby even a study population with 652 workers was not sufficiently large enough to establish the significance of a meaningful association (OR 3.3) (54).

A larger heterogeneity among the studies was observed in the assessment of exposure to physical and psychosocial risk factors. With respect to exposure assessment, only 4 of 14 articles on the influence of force and repetitiveness used the same definition, based on the classical study of Silverstein and her colleagues from 1986 (61). Another important drawback is that 29 studies $(66 \%)$ used questionnaires or interviews to determine the magnitude, frequency, or duration of exposure, and there is ample evidence that self-reports will introduce substantial misclassification in exposure and, thus, considerable attenuation of true associations (62). The heavy reliance on questionnaires for exposure assessment is also reflected in the fact that 20 of the $32(67 \%)$ exposure measures with a significant association with CTS (as reported in table 3) were defined by duration in terms of hours per week or years, which is the exposure dimension with the least random misclassification when estimated by a questionnaire (62).

In about half of the studies $(\mathrm{N}=25,57 \%)$ in this review, the diagnosis of CTS was determined with the use of a questionnaire, a physical examination, or an electrophysiological examination. In the remaining studies $(\mathrm{N}=19,43 \%)$, the diagnosis was based on both symptoms and the results of an electrophysiological examination. The combination of a positive electrodiagnostic study and characteristic symptoms appears to have the best predictive value for a case definition of CTS (4). However, this strict method of diagnosing CTS leads to a low prevalence of the outcome. For example, in a large prospective study by Gerr et al (63), only three prevalent cases, and three incident cases were reported among 630 workers. It is of interest to note that only six studies (14\%) relied on questionnaires only to estimate the presence of CTS, whereas 29 studies (66\%) used questionnaires for exposure assessment. Thus it is reasonable to assume that the heterogeneity among studies is much larger for exposure assessment than for the ascertainment of CTS.

The scores on the methodological quality assessment ranged from 4 to 15 (on a scale from 0 to 16). As a result of a lack of cohort studies, items 12 (follow-up period $\geq 1$ year) and 13 (prospective or retrospective study design) scored positive in only eight articles. Other critical items were "blinding to exposure status" during the verification of CTS $(\mathrm{N}=10)$ and "blinding of case status" during the exposure assessment $(\mathrm{N}=14)$. Blinding to exposure often failed since the physicians were aware of the occupation of the worker under examination, and blinding of CTS status often failed since the diagnostic procedure started with a questionnaire with self-reported exposure measures. The quality assessment of the exposure assessment strategy was restricted to three items, and therefore did not take into account several other issues that are important in the evaluation of exposure assessment, such as measurement error and the contrast in exposure magnitude and duration (64). The methodological quality was not associated with the presence of a significant association between work-related factors and CTS. It is an interesting observation that the methodological quality score of the included articles did not improve over time (figure 2), predominantly due to the publication of various studies in recent years 
that only compare the CTS prevalence across different jobs without any further investigation into underlying exposure patterns and, thus, exposure-response relationships.

Factors that may influence whether a study reports a significant association or not between a physical risk factor and CTS are lack of power, low incidence of outcome, low exposure prevalence, measurement error, and presence of confounding factors. However, the results of our review support the conclusions presented by Palmer et al (9). An excess risk to CTS was reported for assembly workers, meat-processing workers, and food-processing workers. In addition, prolonged use of handheld vibratory tools and prolonged and highly repetitious flexion or extension of the wrist increased the occurrence of CTS. The current review extends existing knowledge with a quantitative assessment of exposure-response relationships between work-related factors and CTS.

A meta-analysis with a pooling of data was considered only for the duration of computer work for $>20$ hours/week $(20,57,59)$ and combined exposure to high force-high repetitiveness (53-55). With regard to computer work, the procedures for case ascertainment differed too much across studies, as was reflected in the prevalence estimates, which varied from $1.4 \%$ (57) to $13.1 \%$ (20). The contradictory findings for computer use and the development of CTS are in agreement with the conclusion of a recent review (65). In two crosssectional studies $(53,54)$, a similar definition of force and repetitiveness was used, but, although significant associations were reported in the separate studies, the large heterogeneity of the risk estimates across studies made the pooling of results not possible. In comparison with other studies on repetitive jobs, it is suggested that harmful cycle times with repetitive movements are $<10$ seconds (42) rather than $<30$ seconds $(48,49)$.

Pooling study results was not possible for exposure to hand-arm vibration and the duration of flexion or extension of the wrist, although several studies have been carried out on these risk factors. With respect to hand-arm vibration, all of the studies consistently reported increased OR values, albeit not always significant associations. A comprehensive exposure assessment strategy was used by Bovenzi and his colleagues (51), who showed that an 8-hour energy-equivalent frequency-weighted acceleration of $3.9 \mathrm{~m} / \mathrm{s}^{2}$ was associated with CTS (51). This value is well above the action value of $2.5 \mathrm{~m} / \mathrm{s}^{2}$ described in the European directive on physical agents (68). The studies on flexion or extension of the wrist show large differences in exposure levels that are already associated with an increased occurrence of CTS, as was found for three case-control studies. Nordstrom et al (52) presented an increased risk of CTS with bending the wrist for $>3$ hours/day, Blanc et al (58) reported hand bending $>2$ hours/day as a risk factor, whereas, in the study of De Krom et al (43), the risk was already elevated when the wrist was extended or flexed for 1 hour/ week. These results, all based on self-reported exposure, are too contradictory for drawing any meaningful conclusion about harmful exposure levels. In addition, the four studies on psychosocial risk factors have presented no indications for an association with CTS.

In summary, this systematic review provides consistent indications that CTS is associated with the following physical risk factors: average requirements for a hand force of $>4$ kilograms, repetitiveness at work (cycle time $<10$ seconds, or $>50 \%$ of a cycle time during which the same movements are performed), and a daily 8-hour energy-equivalent frequency-weighted acceleration of $3.9 \mathrm{~m} / \mathrm{s}^{2}$. Prolonged or repeated flexion and extension of the wrist is a risk factor under scrutiny, but the available evidence does not permit us to provide some guidance on the levels of hazardous exposure levels.

\section{Acknowledgments}

Funding for this project was gratefully received from WorksafeBC, Richmond, Canada (grant RS2006SR05).

\section{References}

1. Bland JD. Carpal tunnel syndrome. BMJ. 2007;335:343-6.

2. de Krom MC, van Croonenborg JJ, Blaauw G, Scholten RJ, Spaans F. Richtlijn 'diagnostiek en behandeling van het carpale-tunnelsyndroom' [Guideline 'diagnosis and treatment of carpal tunnel syndrome']. Ned Tijdschr Geneeskd. 2008;152:76-81.

3. Herbert R, Gerr F, Dropkin J. Clinical evaluation and management of work-related carpal tunnel syndrome. Am J Ind Med. 2000;37:62-74.

4. Rempel D, Evanoff B, Amadio PC, de Krom M, Franklin G, Franzblau A, et al. Consensus criteria for the classification of carpal tunnel syndrome in epidemiologic studies. Am J Public Health. 1998;88:1447-51.

5. de Krom MC, Knipschild PG, Kester AD, Spaans F. Efficacy of provocative tests for diagnosis of carpal tunnel syndrome. Lancet. 1990;335:393-5.

6. Feuerstein M, Miller VL, Burrell LM, Berger R. Occupational upper extremity disorders in the federal workforce: prevalence, health care expenditures, and patterns of work disability. J Occup Environ Med. 1998;40:546-55.

7. Silverstein B, Welp E, Nelson N, Kalat J. Claims incidence of work-related disorders of the upper extremities: Washington state, 1987 through 1995. Am J Public Health. 1998;88:182733.

8. Hagberg M, Morgenstern H, Kelsh M. Impact of occupations and job tasks on the prevalence of carpal tunnel syndrome. Scand J Work Environ Health. 1992;18:337-45. 
9. Palmer KT, Harris EC, Coggon D. Carpal tunnel syndrome and its relation to occupation: a systematic literature review. Occup Med (Lond). 2007;57:57-66.

10. National Institute for Occupational Safety and Health (NIOSH). Musculoskeletal disorders and workplace factors: a critical review of epidemiologic evidence for work-related musculoskeletal disorders of the neck, upper extremity, and low back. Cincinnati (OH): US Department of Health and $\mathrm{Hu}-$ man Sciences, NIOSH; 1997. Publication 97-141.

11. National Research Council. Musculoskeletal disorders and the work place. Washington (DC): The National Academy Press; 2001.

12. Werner RA. Evaluation of work-related carpal tunnel syndrome. J Occup Rehabil. 2006;16:207-22.

13. Bongers PM, Kremer AM, ter Laak J. Are psychosocial factors, risk factors for symptoms and signs of the shoulder, elbow, or hand/wrist?: a review of the epidemiological literature. Am J Ind Med. 2002;41:315-42.

14. van den Heuvel SG, van der Beek AJ, Blatter BM, Hoogendoorn WE, Bongers PM. Psychosocial work characteristics in relation to neck and upper limb symptoms. Pain. 2005;114:4753.

15. Huisstede BM, Miedema HS, van Opstal T, de Ronde MT, Kuiper JI, Verhaar JA, et al. Interventions for treating the posterior interosseus nerve syndrome: a systematic review of observational studies. J Peripher Nerv Syst. 2006;11:101-10.

16. Lievense A, Bierma-Zeinstra S, Verhagen A, Verhaar J, Koes B. Influence of work on the development of osteoarthritis of the hip: A systematic review. J Rheumatol. 2001;28:2520-8.

17. van Tulder M, Furlan A, Bombardier C, Bouter L, Editorial Board of the Cochrane Collaboration Back Review G. Updated method guidelines for systematic reviews in the cochrane collaboration back review group. Spine. 2003;28:1290-9.

18. The Cochrane Collaboration. Dutch cochrane centre [Internet]. Oxford (United Kingdom): The Cochrane Collaboration [updated 26 March 2008; cited 9 July 2008]. Available from: http://www.Cochrane.nl/nl/index.html.

19. Abbas MF, Faris RH, Harber PI, Mishriky AM, El-Shahaly HA, Waheep YH, et al. Worksite and personal factors associated with carpal tunnel syndrome in an Egyptian electronics assembly factory. Int J Occup Environ Health. 2001;7:31-6.

20. Ali KM, Sathiyasekaran BW. Computer professionals and carpal tunnel syndrome (cts). Int J Occup Saf Ergon. 2006;12:319-25.

21. Bovenzi M, Zadini A, Franzinelli A, Borgogni F. Occupational musculoskeletal disorders in the neck and upper limbs of forestry workers exposed to hand-arm vibration. Ergonomics. 1991;34:547-62.

22. Diaz JH. Carpal tunnel syndrome in female nurse anesthetists versus operating room nurses: prevalence, laterality, and impact of handedness. Anesth Analg. 2001;93:975-80.

23. Frost P, Andersen JH, Nielsen VK. Occurrence of carpal tunnel syndrome among slaughterhouse workers. Scand J Work Environ Health. 1998;24:285-92.

24. Jianmongkol S, Kosuwon W, Thumroj E, Sumanont S. Prevalence of carpal tunnel syndrome in workers from a fishnet factory in Thailand. Hand Surg. 2005;10:67-70.

25. Kim JY, Kim JI, Son JE, Yun SK. Prevalence of carpal tunnel syndrome in meat and fish processing plants. J Occup Health. 2004;46:230-4.

26. Kutluhan S, Akhan G, Demirci S, Duru S, Koyuncuoglu HR, Ozturk M, et al. Carpal tunnel syndrome in carpet workers. Int Arch Occup Environ Health. 2001;74:454-7.

27. Leclerc A, Franchi P, Cristofari MF, Delemotte B, Mereau P,
Teyssier-Cotte $\mathrm{C}$, et al. Carpal tunnel syndrome and work organisation in repetitive work: a cross sectional study in France. Occup Environ Med. 1998;55:180-7.

28. Liss GM, Jesin E, Kusiak RA, White P. Musculoskeletal problems among Ontario dental hygienists. Am J Ind Med. 1995;28:521-40.

29. Morgenstern H, Kelsh M, Kraus J, Margolis W. A cross-sectional study of hand/wrist symptoms in female grocery checkers. Am J Ind Med. 1991;20:209-18.

30. Park RM, Nelson NA, Silverstein MA, Mirer FE. Use of medical insurance claims for surveillance of occupational disease: an analysis of cumulative trauma in the auto industry. J Occup Med. 1992;34:731-7.

31. Pocekay D, McCurdy SA, Samuels SJ, Hammond SK, Schenker MB. A cross-sectional study of musculoskeletal symptoms and risk factors in semiconductor workers. Am J Ind Med. 1995;28:861-71.

32. Wang LY, Pong YP, Wang HC, Su SH, Tsai CH, Leong CP. Cumulative trauma disorders in betel pepper leaf-cullers visiting a rehabilitation clinic: experience in Taitung. Chang Gung Med J. 2005;28:237-46.

33. Barnhart S, Demers PA, Miller M, Longstreth WT Jr, Rosenstock L. Carpal tunnel syndrome among ski manufacturing workers. Scand J Work Environ Health. 1991;17:46-52.

34. Bonfiglioli R, Mattioli S, Fiorentini C, Graziosi F, Curti S, Violante FS. Relationship between repetitive work and the prevalence of carpal tunnel syndrome in part-time and fulltime female supermarket cashiers: a quasi-experimental study. Int Arch Occup Environ Health. 2007;80:248-53.

35. Gell N, Werner RA, Franzblau A, Ulin SS, Armstrong TJ. A longitudinal study of industrial and clerical workers: incidence of carpal tunnel syndrome and assessment of risk factors. J Occup Rehabil. 2005;15:47-55.

36. Gorsche RG, Wiley JP, Renger RF, Brant RF, Gemer TY, Sasyniuk TM. Prevalence and incidence of carpal tunnel syndrome in a meat packing plant. Occup Environ Med. 1999;56:417-22.

37. Margolis W, Kraus JF. The prevalence of carpal tunnel syndrome symptoms in female supermarket checkers. J Occup Med. 1987;29:953-6.

38. McCormack JRR, Inman RD, Wells A, Berntsen C, Imbus HR. Prevalence of tendinitis and related disorders of the upper extremity in a manufacturing workforce. J Rheumatol. 1990;17:958-64.

39. Moore JS, Garg A. Upper extremity disorders in a pork processing plant: relationships between job risk factors and morbidity. Am Ind Hyg Assoc J. 1994;55:703-15.

40. Silverstein BA, Hughes RE. Upper extremity musculoskeletal disorders at a pulp and paper mill. Appl Ergon. 1996;27:189_ 94.

41. Lam N, Thurston A. Association of obesity, gender, age and occupation with carpal tunnel syndrome. Aust N Z J Surg. 1998;68:190-3.

42. Roquelaure Y, Mechali S, Dano C, Fanello S, Benetti F, Bureau $\mathrm{D}$, et al. Occupational and personal risk factors for carpal tunnel syndrome in industrial workers. Scand J Work Environ Health. 1997;23(5):364-9.

43. de Krom MC, Kester AD, Knipschild PG, Spaans F. Risk factors for carpal tunnel syndrome. Am J Epidemiol. 1990;132:1102-10.

44. Nathan PA, Istvan JA, Meadows KD. A longitudinal study of predictors of research-defined carpal tunnel syndrome in industrial workers: findings at 17 years. J Hand Surg. 2005;30:593-8. 
45. Nathan PA, Meadows KD, Istvan JA. Predictors of carpal tunnel syndrome: an 11-year study of industrial workers. J Hand Surg [Am]. 2002;27:644-51.

46. Wieslander G, Norback D, Gothe CJ, Juhlin L. Carpal tunnel syndrome (cts) and exposure to vibration, repetitive wrist movements, and heavy manual work: a case-referent study. $\mathrm{Br}$ J Ind Med. 1989;46:43-7.

47. Chiang H-C, Chen S-S, Yu H-S, Ko Y-C. The occurrence of carpal tunnel syndrome in frozen food factory employees. Gaoxiong Yi Xue Ke Xue Za Zhi. 1990;6:73-80.

48. Babski-Reeves KL, Crumtpon-Young LL. Comparisons of measures for quantifying repetition in predicting carpal tunnel syndrome. Int J Ind Ergonom. 2002;30:1-6.

49. Roquelaure Y, Mariel J, Dano C, Fanello S, Penneau-Fontbonne D. Prevalence, incidence and risk factors of carpal tunnel syndrome in a large footwear factory. Int J Occup Med Environ Health. 2001;14:357-67.

50. Latko WA, Armstrong TJ, Franzblau A, Ulin SS, Werner RA, Albers JW. Cross-sectional study of the relationship between repetitive work and the prevalence of upper limb musculoskeletal disorders. Am J Ind Med. 1999;36:248-59.

51. Bovenzi M, Della Vedova A, Nataletti P, Alessandrini B, Poian T. Work-related disorders of the upper limb in female workers using orbital sanders. Int Arch Occup Environ Health. 2005;78:303-10

52. Nordstrom DL, Vierkant RA, DeStefano F, Layde PM. Risk factors for carpal tunnel syndrome in a general population. Occup Environ Med. 1997;54:734-40.

53. Chiang H-C, Ko Y-C, Chen S-S, Yu H-S, Wu T-N, Chang $\mathrm{P}-\mathrm{Y}$. Prevalence of shoulder and upper-limb disorders among workers in the fish-processing industry. Scand J Work Environ Health. 1993;19:126-31.

54. Silverstein BA, Fine LJ, Armstrong TJ. Occupational factors and carpal tunnel syndrome. Am J Ind Med. 1987;11:343-58.

55. Yagev Y, Carel RS, Yagev R. Assessment of work-related risks factors for carpal tunnel syndrome. Isr Med Assoc J. 2001;3:569-71.

56. Cosgrove JL, Chase PM, Mast NJ, Reeves R. Carpal tunnel syndrome in railroad workers. Am J Phys Med Rehabil. 2002;81:101-7.

57. Andersen JH, Thomsen JF, Overgaard E, Ladden CF, Brandt
LP, Vilstrup I, et al. Computer use and carpal tunnel syndrome: a 1-year follow-up study. JAMA. 2003;289:2963-9.

58. Blanc PD, Faucett J, Kennedy JJ, Cisternas M, Yelin E. Selfreported carpal tunnel syndrome: predictors of work disability from the national health interview survey occupational health supplement. Am J Ind Med. 1996;30:362-8.

59. Hou WH, Hsu JH, Lin CH, Liang HW. Carpal tunnel syndrome in male visual display terminal (vdt) workers. Am J Ind Med. 2007;50:1-7.

60. Stevens JC, Witt JC, Smith BE, Weaver AL. The frequency of carpal tunnel syndrome in computer users at a medical facility. Neurology. 2001;56:1568-70.

61. Silverstein BA, Fine LJ, Armstrong TJ. Hand wrist cumulative trauma disorders in industry. Br J Ind Med. 1986;43:779-84.

62. Burdorf A, van der Beek A. Exposure assessment strategies for work-related risk factors for musculoskeletal disorders. Scand J Work Environ Health. 1999;25 suppl 4:25-30.

63. Gerr F, Marcus M, Ensor C, Kleinbaum D, Cohen S, Edwards A, et al. A prospective study of computer users, I: study design and incidence of musculoskeletal symptoms and disorders. Am J Ind Med. 2002;41:221-35.

64. Vlaanderen J, Vermeulen R, Heederik D, Kromhout H, on behalf of the EU Network of Excellence ECNIS Integrated Risk Assessment Group. Guidelines to evaluate human observational studies for quantitative risk assessment. Environ Health Perspect. 2008;116(12):1700-5. doi:101289/ehp11530

65. Thomsen JF, Gerr F, Atroshi I. Carpal tunnel syndrome and the use of computer mouse and keyboard: a systematic review. BMC Musculoskelet Disord. 2008;9:134.

66. Nordander C, Ohlsson K, Balogh I, Rylander L, Palsson B, Skerfving S. Fish processing work: the impact of two sex dependent exposure profiles on musculoskeletal health. Occup Environ Med. 1999;56:256-64.

67. Werner RA, Franzblau A, Gell N, Hartigan AG, Ebersole M, Armstrong TJ. Incidence of carpal tunnel syndrome among automobile assembly workers and assessment of risk factors. J Occup Environ Med. 2005;47:1044-50.

68. Nelson CM, Brereton PF. The European vibration directive. Ind Health. 2005;43:472-9.

Received for publication: 31 July 2008

\section{Appendix I}

\section{Operationalization of the methodological quality assessment}

\section{Criteria}

\section{Study population}

1 Positive if at least 2 of the following 3 items in both groups were reported at baseline:

a. Age [mean (standard deviation or $95 \%$ confidence interval), or dichotomized groups]

b. Gender (number, percentage or both)

c. Sport or leisure-time exposure

2 Positive if the participation of the exposed group and unexposed group was $\geq 70 \%$

3 Positive if the total number of cases was $\geq 50$ 


\section{Assessment of exposure}

Adequate description of the exposure

4 a. Positive if the exposure was clearly defined

$5 \mathrm{~b}$. Positive if the assessment of exposure was described

6 c. Positive if the exposure was assessed by an independent person and was not based on self-reported exposure Assessment of outcome (specific disorder)

Adequate description of the outcome

7 a. Positive if the outcome was clearly defined

8 b. Positive if the method of assessment was suitable

9 c. Positive if the outcome was measured without knowledge of the exposure status by an independent person, thus not based on self-reported symptoms

Study design

10 Positive if the study design was prospective or a retrospective cohort

11 Positive if the inclusion and exclusion criteria were described

12 Positive if the follow-up period was $\geq 1$ year

13 Positive if demographic information was given between completers versus withdrawals

\section{Analysis and data presentation}

14 Positive if risk estimates were presented or when raw data were given which allowed the calculation of risk estimates, such as odds ratios, prevalence ratios or relative risks

Identifying confounders

15 a. Positive if the considered confounders were described

16 b. Positive if the method used to control for confounding was described

\section{Appendix II}

Outcome criteria and assessment and exposure definition and assessment for each included study

\begin{tabular}{|c|c|c|c|c|}
\hline \multirow[t]{2}{*}{ Author } & \multicolumn{2}{|l|}{ Outcome } & \multicolumn{2}{|l|}{ Exposure } \\
\hline & Criteria & Assessment & Definition & Assessment \\
\hline $\begin{array}{l}\text { Margolis \& } \\
\text { Kraus, } 1987 \text { (37) }\end{array}$ & $\begin{array}{l}\text { Self-reported pain in hands or wrists; pain in } \\
\text { wrist or hand that awakened at night; or numb- } \\
\text { ness in hands or wrists or tingling in hands } \\
\text { and fingers }\end{array}$ & Questionnaire & Use of laser scanner (yes; no) & Questionnaire \\
\hline $\begin{array}{l}\text { Silverstein et al, } \\
1987(54)\end{array}$ & $\begin{array}{l}\text { Pain, numbness or tingling in the median nerve } \\
\text { distribution of the hand and nocturnal exac- } \\
\text { erbation and symptoms occurring }>20 \text { times } \\
\text { or lasting }>1 \text { week in the previous year and no } \\
\text { history of acute traumatic onset of symptoms } \\
\text { and of rheumatoid arthritis and onset of symp- } \\
\text { toms in current job and positive Phalen's test } \\
\text { or Tinel's sign and ruling out of cervical root, } \\
\text { thoracic outlet, pronator teres syndromes }\end{array}$ & $\begin{array}{l}\text { Interview, } \\
\text { physical } \\
\text { examination }\end{array}$ & $\begin{array}{l}\text { High force (average hand force require- } \\
\text { ments }>4 \mathrm{~kg} \text { ); repetition (low: cycle time } \\
\text { of }>30 \text { seconds and }<50 \% \text { of cycle time } \\
\text { involved in performance of the same } \\
\text { kind of fundamental cycles; high: cycle } \\
\text { time of }<30 \text { seconds or }>50 \% \text { of the cy- } \\
\text { cle time involved in performance of the } \\
\text { same kind of fundamental cycles) }\end{array}$ & $\begin{array}{l}\text { Jobs were identified by } \\
\text { plant walk-through, pos- } \\
\text { tural data from videotapes } \\
\text { of at least } 3 \text { cycles for } 3 \\
\text { subjects per job, electro- } \\
\text { myographic recordings } \\
\text { used to estimate require- } \\
\text { ments for hand force }\end{array}$ \\
\hline $\begin{array}{l}\text { Wieslander et al, } \\
1989(46)\end{array}$ & $\begin{array}{l}\text { Diagnosis by hand surgeon and confirmed } \\
\text { electroneurographically by measurement of the } \\
\text { conduction velocities in the median nerve at the } \\
\text { wrist level }\end{array}$ & $\begin{array}{l}\text { Physical ex- } \\
\text { amination, } \\
\text { electrodi- } \\
\text { agnostic } \\
\text { examination }\end{array}$ & $\begin{array}{l}\text { Duration of use of vibrating tools (year); } \\
\text { duration of repetitive movement of wrist } \\
\text { (year); duration of work causing great } \\
\text { load on wrist (year) }\end{array}$ & Interview by telephone \\
\hline
\end{tabular}




\begin{tabular}{ll}
\hline Author & Outcome \\
\cline { 2 - 3 } & Criteria \\
\hline Chiang et al, & $\begin{array}{l}\text { Symptoms of numbness, pain or tingling in } \\
\text { the fingers innervated by the median nerve and } \\
\text { onset of CTS since working on current job and } \\
\text { positive Tinel's sign or Phalen's test and no } \\
\text { evidence of CTS-related systemic diseases or } \\
\text { injuries }\end{array}$
\end{tabular}

de Krom et al, Tingling, pain, numbness or a combination of 1990 (43) the three in the fingers innervated by the median nerve with a frequency of twice a week or more, which in most cases woke the patient up at night, and confirmation in electrodiagnostic examination

McCormack et al, Positive Tinel's sign and Phalen's test con1990 (38) firmed by physician

Barnhart et al, Electrophysiological criterion (increased median 1991 (33) nerve latency of at least $0.5 \mathrm{~ms}$ ) and Phalen's or Tinel's sign or ever having had hand pain, tingling, numbness or nocturnal hand pain or a combination of the symptoms.

Bovenzi et al, Pain, numbness or tingling in the median nerve 1991 (21) distribution of the hand, positive Tinel's sign or
Phalen's test, diminished sensitivity to touch or pain in three-and-a-half fingers on the radial side of the hand, weakness in pinching or gripping

Morgenstern et Pain in the hands or wrists or nocturnal pain in $\mathrm{al}, 1991(29) \quad$ the wrists or hands that awakened the patients or numbness in the hands or fingers, or tingling in hands of fingers

Park et al, 1992 CTS in insurance claim

(30)

Chiang et al, 1993 (53)

Symptoms of numbness, pain, or tingling in the fingers innervated by the median nerve and onset of CTS since working on current job and positive Tinel's sign or Phalen's test and no evidence of CTS-related systemic diseases or injuries

Moore, \& Garg, Presence of numbness or tingling in the hands 1994 (39) and electrodiagnostic confirmation

Liss et al, 1995 Presence of CTS being told by care providers (28)

Pocekay et al, Physician diagnosed CTS in the past year 1995 (31)

Blanc et al, 1996 Self-reported condition affecting the wrist and (58)

Silverstein \&

Hughes, 1996

(40) hand called CTS

Symptoms of pain, numbness or tingling in the hand more than once or lasting $>1$ week in past 12 months and positive Phalen's test or Tinel's sign in addition to a negative Spurling test and a negative hyperabduction test

Nordstrom et al, Diagnosis of CTS by a physician or any explicit 1997 (52) treatment for CTS and numbness, tingling, pain, or paraesthesia in the hand, wrist, arm or forearm within 1 month of the date of the CTS diagnosis

Roquelaure et al, At least 3 of the following conditions: tingling, 1997 (42) pain, or numbness in the median nerve distribution of the hand with nocturnal exacerbation with $>20$ occurrences or lasting $>3$ weeks in the previous year, a positive Tinel's sign and a positive Phalen's test or hypoesthesia in the region of the median nerve, slowing of the sensory or motor conduction velocities $(<40 \mathrm{~m} / \mathrm{s})$ in the median nerve at the wrist level, or surgical release of the transverse carpal ligament

Exposure

Assessment

Interview, neurological screening and electrodiagnostic examination

Questionnair electrodiagnostic examination

Physical examination

Definition

Cold [handling frozen foods with temperature between -12 to $-15^{\circ} \mathrm{C}$ (yes;

no)]; repetition (low: cycle time of $>30$ seconds and $<50 \%$ of cycle time involved in performance of the same kind of fundamental cycles; high: cycle time of $<30$ seconds or $>50 \%$ of the cycle time involved in performance of the same kind of fundamental cycles)

Questionnaire, Repetitive jobs (repeated or sustained physical exam- activities that involved flexion, extensio ination, electrodiagnostic examination or ulnar deviation of the wrist by $45 \mathrm{de}-$ grees or both; radial deviation $>30$ degrees; or use of pinch type grip)

Interview and Hand-arm vibration $\left(\mathrm{m} / \mathrm{s}^{2}\right)$ complete neurological and orthopedic assessment by physiatrist

Questionnaire

\section{Q}

Medical records

Interview, neurological screening and electrodiagnostic examination

Interview, electrodiagnostic examination

Duration of work as checker (hours/ week, year)

Job title

High repetitive jobs (cycle time of $<30$ seconds or $>50 \%$ of the cycle time involved in performance of the same type of fundamental cycles); high force jobs (an estimated average hand force of $>3 \mathrm{~kg}$ )

Hazardous jobs (yes; no), based on 8 potential risk factors for CTS

Questionnaire Job title

Questionnaire Job title

Questionnaire

Interview,

physical

examination

Repetitive hand bending $>2$ hours/day (yes, no)

Job title

Medical record Duration of use of power tools or machinery (hours/day); duration of bending hands or wrists (hours/day); job control (7 items with ordinal scale of 1 to 5 )

Medical records based on interview, examination and electrodiagnostic examination High force [load was $>1 \mathrm{~kg}$ with a fre-

High force [load was $>1 \mathrm{~kg}$ with a fre-
quency of exertion in excess of 10 times/
the parts handled, dire hour (yes; no)]; work cycle [ $<10$ seconds (yes; no)]; changes in activity or breaks $<15 \%$ of worktime (yes: no); no job rotation (yes; no)
Assessment

Job analyses made by the industrial hygienist of the frozen food factory

Expert opinion based on job title

Job analyses made by the industrial hygienist

Measurements of chain saws

Questionnaire

Expert opinion based on job title

Jobs were identified by plant walk-through, postural data from videotapes per job, electromyographic recordings used to estimate hand force per job Jobs identified by plant walk-through, postural data from videotapes per job, hand-force measurements

Questionnaire

Questionnaire

Questionnaire

Interview and video analyses

Telephone interview observation 


\begin{tabular}{|c|c|c|c|c|}
\hline \multirow[t]{2}{*}{ Author } & \multicolumn{2}{|l|}{ Outcome } & \multicolumn{2}{|l|}{ Exposure } \\
\hline & Criteria & Assessment & Definition & Assessment \\
\hline $\begin{array}{l}\text { Frost et al, } 1998 \\
\text { (23) }\end{array}$ & $\begin{array}{l}\text { A combination of symptoms, occurring at least } \\
1 \text { night a week and current symptoms involv- } \\
\text { ing at least } 1 \text { of } 3 \text { radial fingers, and positive } \\
\text { neurophysiological criteria or previous opera- } \\
\text { tion for CTS }\end{array}$ & $\begin{array}{l}\text { Questionnaire, } \\
\text { physical and } \\
\text { electrodi- } \\
\text { agnostic } \\
\text { examination }\end{array}$ & Job title & Questionnaire \\
\hline $\begin{array}{l}\text { Lam \& Thurston, } \\
1998(41)\end{array}$ & $\begin{array}{l}\text { Diagnosis on the basis of history and clinical } \\
\text { examination and confirmed by a nerve conduc- } \\
\text { tion study }\end{array}$ & $\begin{array}{l}\text { Questionnaire, } \\
\text { physical exam- } \\
\text { ination, elec- } \\
\text { trodiagnostic } \\
\text { examination }\end{array}$ & $\begin{array}{l}\text { Duration of keyboard use (long; short); } \\
\text { heavy manual work (low; medium; high) }\end{array}$ & Questionnaire \\
\hline $\begin{array}{l}\text { Leclerc et al, } \\
1998(27)\end{array}$ & $\begin{array}{l}\text { Positive Tinel's sign or Phalen's test or if a } \\
\text { definite diagnosis based on nerve condition ve- } \\
\text { locity had been established before the medical } \\
\text { examination }\end{array}$ & $\begin{array}{l}\text { Questionnaire, } \\
\text { physical } \\
\text { examination }\end{array}$ & Jobs with repetitive work (yes; no) & $\begin{array}{l}\text { Jobs assigned by an } \\
\text { ergonomist, based on a } \\
\text { questionnaire }\end{array}$ \\
\hline $\begin{array}{l}\text { Gorsche et al, } \\
1999(36)\end{array}$ & $\begin{array}{l}\text { History of pain and numbness along the median } \\
\text { nerve distribution of the hand lasting }>1 \text { week } \\
\text { at the time of the examination and a positive } \\
\text { Tinel's sign or a positive Phalen's test }\end{array}$ & $\begin{array}{l}\text { Interview, } \\
\text { physical } \\
\text { examination }\end{array}$ & Tool use (yes; no) & Interview \\
\hline $\begin{array}{l}\text { Latko et al, } 1999 \\
(50)\end{array}$ & $\begin{array}{l}\text { Positive hand diagram and difference in peak } \\
\text { latency of } 0.5 \mathrm{~ms} \text { between the ulnar and median } \\
\text { nerves }\end{array}$ & $\begin{array}{l}\text { Questionnaire, } \\
\text { electrodi- } \\
\text { agnostic } \\
\text { examination }\end{array}$ & $\begin{array}{l}\text { Repetitive work (visual analogue scale } \\
\text { of 0-10 cm) (low: 0-3.3 cm; medium: } \\
3.3-6.6 \mathrm{~cm} \text {; high: } 6.6-10 \mathrm{~cm} \text { ) }\end{array}$ & $\begin{array}{l}\text { Visual analogue scale } \\
(0-10 \mathrm{~cm}) \text { for repetition }\end{array}$ \\
\hline $\begin{array}{l}\text { Nordander et al, } \\
1999(66)\end{array}$ & $\begin{array}{l}\text { Pain before provocation and palpation of the } \\
\text { tissues and complaints in the neck and upper } \\
\text { limbs during the past } 12 \text { months and past } 7\end{array}$ & $\begin{array}{l}\text { Interview, } \\
\text { physical } \\
\text { examination }\end{array}$ & Job title & $\begin{array}{l}\text { Questionnaire, ergonomic } \\
\text { workplace analysis }\end{array}$ \\
\hline
\end{tabular}

Abbas et al, 2001 Pain or paresthesia or both in the median nerve Interview,

(19) distribution and a decrease in the NCV of $42 \mathrm{~ms}$ or more or a delay of $>4.2$ seconds and confirmed by a physician or both

physical exam- (yes; no) intermediate versus power ination, electrodiagnostic examination (yes; no)]

Grip assigned at job title level by industrial hygienist in a walk-through

Diaz, 2001 (22) History of surgical median nerve decompres- Questionnaire, Job title sion or a combination of nocturnal hand pain, a physical positive hand pain diagram, positive Tinel's sign examination and Phalen's test

Kutluhan et al, A distal latency difference of greater than 0.5 2001 (26) ms between the median and ulnar sensory nerves

Roquelaure et al, Presence of paresthesia, pain, or numbness 2001 (49) affecting at least part of the median nerve distribution of the hand occurring for at least 1 week or, if intermittent, occurring at least 10 times during the previous 12 months, positive Tinel's sign or Phalen's test or diminished or absent sensation to pin prick in the median nerve distribution, and absence of other causes of hand numbness or paresthesia.

Stevens et al, Pins and needles sensations or numbness in 2001 (60) the hands and orthodromic median midpalma latency of $>2.2$ ms or a median-ulnar palmar latency difference of $>0.4 \mathrm{~ms}$ using a distance of $8 \mathrm{~cm}$ and $>2$ positive responses to 4 times: awakened from sleep by paresthesia, or hand goes to sleep during driving, hand goes to sleep during reading or relief by shaking hand.

Electro-

diagnostic examination

Questionnaire, Job analyses [work cycle time $<30 \mathrm{sec}-$ interview

and physical examination onds (yes; no); wrist deviation (yes; no); rapid trigger movements (yes; no); vibration transmitted to the hand (yes; no); wrist flexion > 45 degrees (yes; no); wrist extension $>45$ degrees (yes; no)]: questionnaire [permanent time pressure (yes; no); few possibilities to take breaks (yes; no); work strongly controlled by superiors (yes; no); high work demands (yes; no); low task control (yes; no); low social support (yes; no)

Questionnaire, Mouse use (no; occasionally; frequentelectrodiagnostic ly); current typewriter use (yes; no)

Questionnaire

Interview

Questionnaire and job analyses by observations at the workplace examination

Yagev et al, 2001 Sensory latency longer than 3.9 ms or motor (55) latency longer than $4.5 \mathrm{~ms}$ or both

Electrodiagnostic examination

High repetitive jobs (a cycle time of $<30$ seconds or $>50 \%$ of the cycle time involved in performance of the same type of fundamental cycles); high force jobs (an estimated average hand force $>4 \mathrm{~kg})$

Babski-Reeves Positive for Phalen's sign or the carpal com\& Crumtponpression test and pain, numbness, or tingling Young, 2002 (48) in the median nerve distribution of the hand or wrist
Questionnaire, Repetition: cycle time $<30$ seconds physical examination
Questionnaire

Observation and video assessment for $30 \mathrm{cy}$ cles for each hand per participant 


\begin{tabular}{|c|c|c|c|c|}
\hline \multirow[t]{2}{*}{ Author } & Outcome & & Exposure & \\
\hline & Criteria & Assessment & Definition & Assessment \\
\hline
\end{tabular}

Cosgrove et al, Positive Tinel's sign and Phalen's test, or thenar Physical High force (average hand force require- Written job analysis,

2002 (56) atrophy examination

ment of $>4 \mathrm{~kg}$ ); repetition (low: cycle time of $>30$ seconds and $<50 \%$ of the cycle time involved in performance of the same kind of fundamental cycles; high: cycle time of $<30$ seconds or $>50 \%$ of the cycle time involved in performancd of the same kind of fundamental cycles)

Nathan et al, Abnormal nerve conduction (latency differ2002 (45) ence $\geq 0.40 \mathrm{~ms}$ ), confirmed by two or more specific CTS symptoms (numbness, tingling, or nocturnal awakening) or one specific symptom and two or more nonspecific symptoms (pain tightness, or clumsiness) present two or more times per month or a history of carpal tunnel release surgery

Andersen et al, Tingling or numbness at night at least once a 2003 (57) week within the last 3 months in the areas of the right hand innervated by the median nerve

Kim et al, 2004 Symptom in the fingers and the wrist that con(25) tinued for $>1$ week or more than once a month during the last year with positive Tinel's sign, Phalen's test or NCS

Bovenzi et al 2005 (51)

Gell et al, 2005 (35)

Numbness, tingling, burning, or pain in at leas two of digits 1, 2, 3 and nocturnal symptoms and positive Tinel's sign or Phalen's test

Diagnosis made by physician or numbness, tingling, burning or pain in the distribution of the median nerve with ipsilateral median nerve conduction slowing (difference of $\geq 0.5 \mathrm{~ms}$ between the ipsilateral ulnar and median sensory peak latency)

Jianmongkol et Pain, paresthesia or sensory loss along the meal, 2005 (24) dian nerve distribution and positive for Tinel's sign or Phalen's test or nocturnal exacerbation of symptoms or motor loss with wasting of abductor pollicis brevis or abnormal nerve conduction times

Nathan et al, Abnormal nerve conduction (latency difference 2005 (44) $\geq 0.40 \mathrm{~ms}$ ) confirmed by $\geq 2$ specific CTS symptoms (numbness, tingling or nocturnal awakening) or one specific symptom and $\geq 2$ nonspecific symptoms (pain, tightness or clumsiness) present $\geq 2$ times per month, or a history of carpal tunnel release surgery

Wang et al, 2005 Pain and paraesthesia at the wrist, hand and (32) fingers, compatible with the sensory distribution of the median nerve, and a positive Phalen test or the cross-sectional area of the median nerve at the level of the pisiform bone on ultrasonography was $>0.11 \mathrm{~cm}^{2}$ in combination with compression sign on an longitudinal scan

Werner et al, Numbness, tingling, burning or pain in the 2005 (67) median distribution and a median sensory-peak latency difference of $\geq 0.5 \mathrm{~ms}$ compared with the ipsilateral ulnar sensory peak latency

\section{ak phy}

Questionnaire, High social support (yes; no)

Questionnaire, Repetition (1 to 5 Likert scale); heavy electrodi-

agnostic

examination

lifting (1 to 5 Likert scale); keyboard use

(1 to 5 Likert scale); force (1 to 5 Likert

scale); hand-arm vibration (yes; no)

Questionnaire, Duration of right-handed mouse use

interview

(hours/week); duration of keyboard use (hours/week); high job demands (yes; no); low job control (yes; no); low social support (yes; no); time pressure (yes; no)

Questionnaire, Repetitive movements (yes; no)

physical exam-

ination, elec-

trodiagnostic

examination

Interview,

physical

Hand-arm vibration $\left(\mathrm{m} / \mathrm{s}^{2}\right)$

examination

Questionnaire, Job title

physical exam-

ination, elec-

trodiagnostic

examination

Questionnaire, Job title

interview,

physical

examination

Questionnaire, Repetition (1 to 5 Likert scale); heavy

electrodi-

agnostic

lifting (1 to 5 Likert scale); force (1 to 5

Likert scale)

Questionnaire, Job title

physical exam-

ination, ultra-

sonography

physical exam-

ination, elec-

trodiagnostic

examination

Ali \&

Sathiyasekaran

Pain or numbness on the anterior surface of the $2006(20)$

Bonfiglioli et al, 2007 (34)

index, middle or radial half of the ring finger for the past week and a Tinel's sign or Phalen's test

Nocturnal or diurnal numbness or both, tin-

physical

examination

Hand position (neutral; flexed; extended); duration of computer work (hours/day); duration of computer work (years)

Questionnaire, Job title

Questionnaire

videotaped job analysis,

deposition transcripts and interview

Observation

Assigned by job title

Direct measurements on orbital sanders

Expert opinion

Interview

Observation

first 3 fingers within the last month, and abnor- agnostic

mal nerve conduction

examination

Hou et al, 2007 Symptoms in upper extremities
Questionnaire
Observation questionnaire

Questionnaire

Questionnaire
Duration of keyboard use (hours/day) duration of mouse use (hours/day) 